\title{
A systematic review and narrative synthesis of data-driven studies in schizophrenia symptoms and cognitive deficits
}

Tesfa Dejenie Habtewold (10 1,2, Lyan H. Rodijk ${ }^{1,3}$, Edith J. Liemburg², Grigory Sidorenkov ${ }^{1}$ H. Marike Boezen ${ }^{1}$, Richard Bruggeman ${ }^{2,4}$ and Behrooz Z. Alizadeh (1) ${ }^{1,2}$

\begin{abstract}
To tackle the phenotypic heterogeneity of schizophrenia, data-driven methods are often applied to identify subtypes of its symptoms and cognitive deficits. However, a systematic review on this topic is lacking. The objective of this review was to summarize the evidence obtained from longitudinal and cross-sectional data-driven studies in positive and negative symptoms and cognitive deficits in patients with schizophrenia spectrum disorders, their unaffected siblings and healthy controls or individuals from general population. Additionally, we aimed to highlight methodological gaps across studies and point out future directions to optimize the translatability of evidence from data-driven studies. A systematic review was performed through searching PsycINFO, PubMed, PsycTESTS, PsyCARTICLES, SCOPUS, EMBASE and Web of Science electronic databases. Both longitudinal and cross-sectional studies published from 2008 to 2019, which reported at least two statistically derived clusters or trajectories were included. Two reviewers independently screened and extracted the data. In this review, 53 studies (19 longitudinal and 34 cross-sectional) that conducted among 17,822 patients, 8729 unaffected siblings and 5520 controls or general population were included. Most longitudinal studies found four trajectories that characterized by stability, progressive deterioration, relapsing and progressive amelioration of symptoms and cognitive function. Cross-sectional studies commonly identified three clusters with low, intermediate (mixed) and high psychotic symptoms and cognitive profiles. Moreover, identified subgroups were predicted by numerous genetic, sociodemographic and clinical factors. Our findings indicate that schizophrenia symptoms and cognitive deficits are heterogeneous, although methodological limitations across studies are observed. Identified clusters and trajectories along with their predictors may be used to base the implementation of personalized treatment and develop a risk prediction model for high-risk individuals with prodromal symptoms.
\end{abstract}

\section{Introduction}

In psychiatry, phenotypic heterogeneity of disorders and their overlapping symptoms that may presumably share some fundamental biologic underpinnings is a major

\footnotetext{
Correspondence: Tesfa Dejenie Habtewold (tesfadej2003@gmail.com) or Behrooz Z. Alizadeh (b.z.alizadeh@umcg.nl)

${ }^{1}$ Department of Epidemiology, University Medical Center Groningen, University of Groningen, Groningen, The Netherlands

${ }^{2}$ Department of Psychiatry, Rob Giel Research Center, University Medical

Center Groningen, University Center for Psychiatry, University of Groningen, Groningen, The Netherlands
}

Full list of author information is available at the end of the article challenge for tailoring individualized therapies ${ }^{1}$. Similarly, the course and phenotypic expression of schizophrenia are variable ${ }^{2}$. Schizophrenia is a complex polygenic psychotic disorder with a lifetime morbidity risk of $0.7 \%^{3}$. The twin- and SNP-based heritability estimate of schizophrenia was $80 \%^{4}$ and $30 \%{ }^{5}$, respectively.

According to the diagnostic and statistical manual of mental disorders (DSM) criteria, the clinical manifestations of schizophrenia are positive (e.g., hallucinations, delusions and disorganized behaviour) and negative (e.g., emotional expressive deficit, social amotivation, social

\section{(c) The Author(s) 2020}

(c) Open Access This article is licensed under a Creative Commons Attribution 4.0 International License, which permits use, sharing, adaptation, distribution and reproduction in any medium or format, as long as you give appropriate credit to the original author(s) and the source, provide a link to the Creative Commons license, and indicate if changes were made. The images or other third party material in this article are included in the article's Creative Commons license, unless indicated otherwise in a credit line to the material. If material is not included in the article's Creative Commons license and your intended use is not permitted by statutory regulation or exceeds the permitted use, you will need to obtain permission directly from the copyright holder. To view a copy of this license, visit http://creativecommons.org/licenses/by/4.0/. 
withdrawal and difficulty in experiencing pleasure) symptoms ${ }^{6}$. Cognitive deficit is also one of the hallmark manisfestations of schizophrenia that occur in $75-80 \%$ of patients and often associated with poor daily functioning and quality of life ${ }^{7}$. Cognitive impairment in schizophrenia can be selective or general though the most common deficits occur in executive function, processing speed, memory (e.g. episodic, verbal and working), attention, verbal fluency, problem-solving and social cognition $^{8-11}$. Patients harbor a wide range of subjectively defined symptoms, which together yields instinctively heterogeneous groups of people who are collectively diagnosed with schizophrenia. Subclinical or prodromal symptoms are also evident in relatives of patients with schizophrenia and healthy general population ${ }^{12-14}$.

Despite a century of efforts, understanding the heterogeneity in the clinical presentation and course of schizophrenia has been unsuccessful. This can be due to the subjective measurement of its clinical symptoms, variation in response to treatment, lack of valid, stable, and meaningful sub-phenotyping methods, and molecular complexity with limited understanding of the pathophysiology $^{15-17}$. Phenotypic heterogeneity can be related to several intrinsic and extrinsic factors and expressed in patients, time, and disease sub-phenotypes ${ }^{16,18}$. Identification of meaningful homogeneous subgroups of people based on their symptoms or endophenotypes (e.g. neuropsychological markers, neural substrates, and neurological soft signs) requires the use of both supervised and unsupervised analyses. Distinguishing heterogeneous patients to more behaviorally and biologically similar subgroups is expedient not only to unveil common etiologies but also to examine the patterns of clinical symptoms, understand the biology of disease, predict treatment response and develop a new targeted treatment that improves recovery and functional outcomes ${ }^{15,16,19,20}$.

For tackling heterogeneity, in the past decade, numerous efforts have been undertaken by carefully designing studies and developing statistical models implemented in various programming languages and software ${ }^{16}$. In 2013, the American Psychiatric Association also endorsed a dimensional approach to identify intermediate categories based on the subjective report of severity of symptoms ${ }^{6}$. As a result, researchers have been using latent class cluster analyses and growth mixture models to explore clusters of individuals and trajectories of clinical symptoms in various settings ${ }^{15,21,22}$. Statistical methods can be used to identify subgroups and describe within and between individual variations to guide clinicians and statisticians to explore the relationship of diseases with various clinical and functional outcomes, treatment response, and neuropathological change. Moreover, subtyping using imaging, biological and symptom data is a recognizable method and widely used in psychiatric research $^{21}$.
Several reviews have been conducted on positive symptoms ${ }^{23}$, negative symptoms ${ }^{24-26}$ and cognitive dysfunction ${ }^{7,9,27-35}$. However, these reviews have largely focused on the conventional approach for determining an average change in the course of symptoms over time and the difference between subjects (e.g., patient vs sibling, sibling vs control, or patient vs control) and diagnosis. Reviewed studies are also based on correlation analysis, which is believed not to be a strong measure of association between predictors and outcomes ${ }^{36}$. Besides, these primary studies vary in terms of study population and use of assessment tools, scoring and standardization techniques, and have several limitations, such as small sample size, short duration of follow-up and limited use of data from healthy siblings and/or controls ${ }^{9,37,38}$. Of interest, none of these reviews fully addressed evidence from both longitudinal and cross-sectional data-driven studies on schizophrenia symptoms and cognitive deficits among patients with schizophrenia spectrum disorders, relatives and healthy controls. Taken together, thus far, our understanding of the heterogeneity of the course of schizophrenia symptoms and cognitive deficits is still limited. In the present systematic review, we summarized the contemporary evidence from cross-sectional and longitudinal studies on positive and negative symptoms and cognitive deficits among patients with schizophrenia spectrum disorders, their unaffected siblings and healthy people. Additionally, we explored the extent and origin of heterogeneity across studies. We further highlighted common methodological gaps and point out future directions to optimize the translatability of evidence from data-driven studies within the outlook of a personalized approach.

\section{Methods \\ Registration and reporting}

This systematic review was conducted and reported based on a registered protocol ${ }^{39}$ and the Preferred Reporting Items for Systematic Review and Meta-Analysis (PRISMA) statement (Supplementary File 1), respectively $^{40,41}$. The screening and selection process of the reviewed articles are further illustrated using a PRISMA flow diagram.

\section{Databases and search terms}

A systematic search of PubMed, PsycINFO, PsycTESTS, PsycARTICLES, SCOPUS, EMBASE and Web of Science electronic databases was performed. A comprehensive search strategy was developed for PubMed and adapted for each database in consultation with a medical information specialist (Supplementary File 2). The following search terms were used in their singular or plural form in the title, abstract, keywords and text fields of the articles: "schizophrenia", "psychosis", "non-affective psychosis", "cognitive deficit", "cognitive dysfunction", "cognitive 
alteration", "negative symptoms", "deficit syndrome", "positive symptoms", "psychopathology", "cognit", "neuropsycholog*", "neurocognition", "longitudinal", "followup", "course", "heterogeneity", "endophenotype", "profile", "cluster analysis", "siblings", "healthy controls", "latent class analyses", "Symptom trajectories", "traject", "group modelling" and "trajectory". Cross-references of included articles and grey literature were also hand-searched. Furthermore, we searched the table of contents of the journals of Schizophrenia Research, Schizophrenia Bulletin, Acta Psychiatrica Scandinavica, BMC Psychiatry, American Journal of Psychiatry and British Journal of Psychiatry to explore relevant studies. The freezing date for the final search was August 2019. In this review, we use 'trajectory' for groups identified in longitudinal studies and "cluster" for groups identified in cross-sectional studies.

\section{Inclusion and exclusion criteria}

Studies which met the following criteria were included: (1) longitudinal and cross-sectional studies; (2) studies that reported at least two clusters or trajectory groups of individuals using a statistical method based on a distinct positive symptom, negative symptom, and cognitive deficit or a combination of these symptoms; (3) studies conducted in patients with schizophrenia spectrum disorders, unaffected relatives, or healthy individuals irrespective of their clinical (e.g. medication status, severity of illness) and sociodemographic characteristics; and (4) studies published in English from 2008 to 2020. The publication year was limited to the last decade to capture the latest available evidence, which is likely to provide statistically powerful estimates and successfully subtyping schizophrenia symptoms given the increased number of large cohorts. To maximize the number of searched articles, the follow-up period in longitudinal studies was not restricted. Longitudinal studies based on the analyses of the mean levels of change of symptom scores were excluded because they did not capture individuals' patterns of change over time by treating between-subject variation as an error, so that the actual heterogeneity of groups cannot be revealed ${ }^{42}$. Also, studies based on the non-statistical methods of clustering (e.g. family-based clustering) were excluded. Review papers, commentaries, conference abstracts, duplicate studies, editorials, and qualitative studies were excluded as well. Furthermore, we excluded studies in which the trajectory groups or clusters were generated based on scores constructed using a combination of schizophrenia symptoms and other unspecified psychotic symptoms.

\section{Data retrieval and synthesis}

Studies retrieved from all databases were exported to RefWorks version 2.0 for Windows web-based citation manager, which followed by the removal of close and exact duplicates. All independent studies were exported to a Microsoft Excel spreadsheet to screen for further inclusion criteria. Authors T.D.H. and L.H.R. independently screened the titles and abstracts. The two reviewers had a substantial agreement (Kappa statistic $(\kappa)=0.62$ ). Inconsistent decisions were discussed and solved with consensus. Finally, full-text was reviewed, and the following data were independently extracted by T.D.H. and L.H.R.: first author name, publication year, country, cohort/research center, study population, sample size, symptom dimension(s), assessment tool, study design, duration of follow-up for longitudinal studies, frequency of assessment, method of calculating composite score, method of clustering/trajectory analysis, number of identified clusters or trajectory groups and significant correlates of clusters and predictors of trajectories ${ }^{43}$. The corresponding author was contacted by email if the full-text of included article was not accessible. When studies did not report the cohort or research center, we extracted the institutional affiliation of the first or corresponding author.

\section{Results \\ Search results}

In total, 2262 articles were identified through database searching and an additional 26 articles were obtained through manual searching of cross-references and tables of content of relevant journals. After removing duplicate and unrelated articles, the titles and abstracts of 1292 articles were screened. The evaluation of titles and abstracts resulted in the exclusion of 1231 articles. In total, 61 articles were selected for full-text review, and eight articles ${ }^{4-51}$ were excluded due to unclear outcomes, mixed diagnosis of the study population and use of a nonstatistical method of clustering or clustering based on different phenotypes of schizophrenia. Finally, data were extracted from 53 longitudinal and cross-sectional studies. The PRISMA flow diagram of screening and the selection process is shown in Fig. 1.

\section{Overview of included studies}

The included 53 studies were conducted globally in 30 countries and published over a decade from 2009 to 2020. Seventeen studies were conducted in the USA and few studies were internationally conducted. Of these, $19 \mathrm{stu}$ dies were longitudinal that involved 11,684 patients, 1059 siblings and 2194 controls or general population from more than eight countries, whereas 34 studies were cross-sectional that involved 6138 patients, 7670 siblings, and 3326 controls from 14 countries. Most of the longitudinal studies examined trajectories of positive and negative symptoms in patients, whereas most of the crosssectional studies explored cognitive subtypes in patients. Only one longitudinal study ${ }^{52}$ and three cross-sectional 


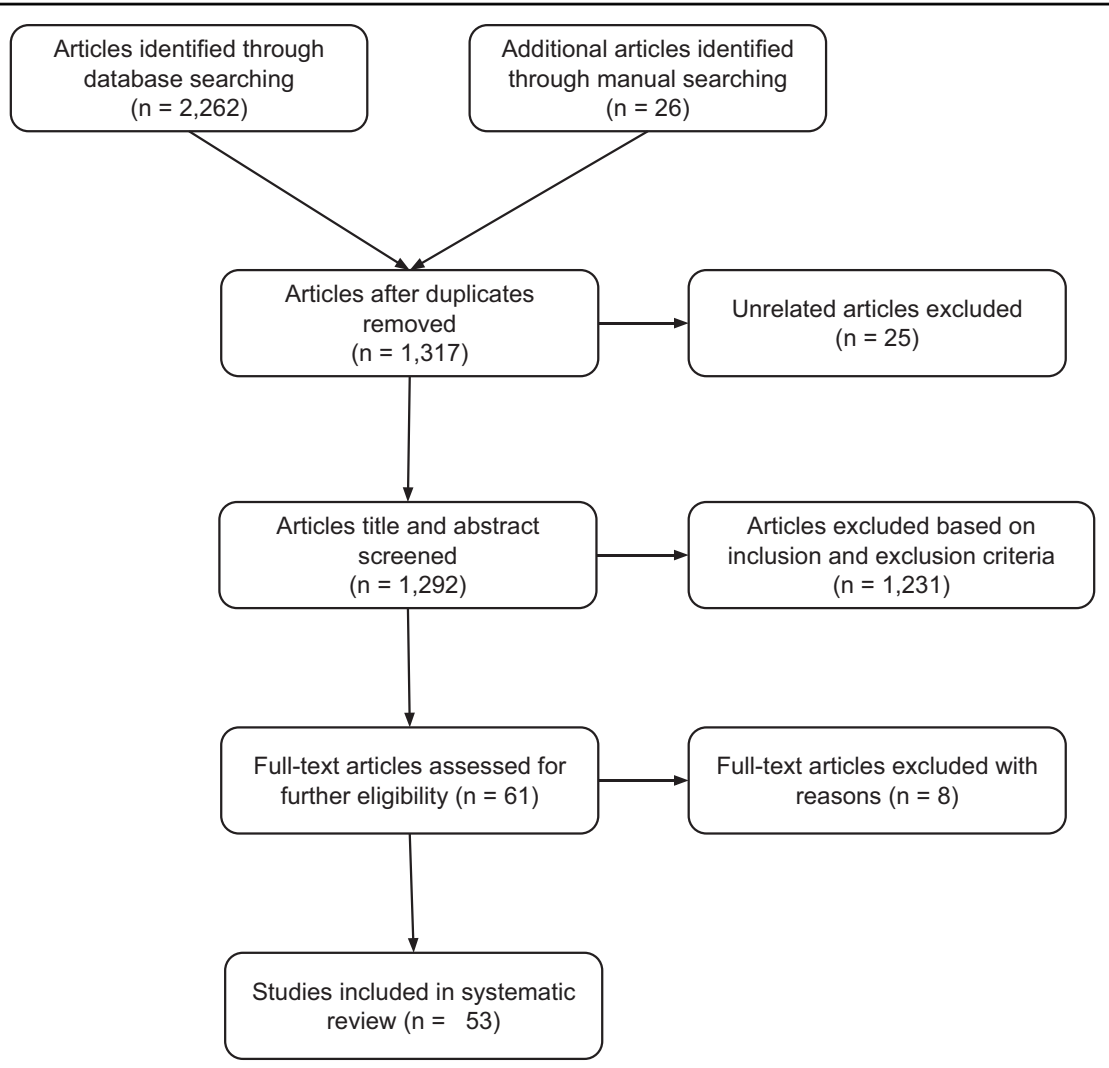

Fig. 1 PRISMA flow diagram illustrating the screening and selection of literature.

studies $^{53-55}$ examined cognitive subtypes among siblings. Overall, two to six subtypes of positive and negative symptoms and cognitive deficits were identified.

\section{Longitudinal studies}

In total, 19 longitudinal studies were reviewed that included all population age groups with the duration of follow-up ranged from six weeks to 10 years. The sample size ranged from 138 to 1990 subjects. Even though all studies had a similar aim, they have used slightly different models of trajectory analysis and model selection criteria. Growth mixture modelling $(\mathrm{GMM})^{17,56,57}$, latent class growth analysis (LCGA) ${ }^{16,19,20,58-61}$, mixed-mode latent class regression modelling ${ }^{22,62,63}$, group-based trajectory modelling $(\mathrm{GBTM})^{52,64-66}$ and Ward's method ${ }^{67}$ were reported data-driven methods. Akaike's Information Criterion (AIC), Bayesian information criterion (BIC) (i.e., used in most studies), deviance information criterion (DIC), logged Bayes factor, sample size adjusted BIC (aBIC), bootstrap likelihood ratio test [BLRT], Gap statistic, Lo-Mendell-Rubin Likelihood Ratio Test (LMRLRT) and entropy were reported model selection indices.

Most longitudinal studies, Table 1 , investigated the trajectory of positive, negative or both symptoms in patients whereas one study ${ }^{68}$ explored the trajectory of schizotypy in a nonclinical population. Another study ${ }^{57}$ examined the association between positive and negative symptom trajectories in patients. Moreover, three studies examined the long-term trajectories of cognitive impairment in patients, their unaffected siblings and healthy controls ${ }^{16,52,66}$. One study ${ }^{52}$ investigated the association between patients' and siblings' cognitive trajectories as well. Overall, these studies characterized the general pattern of identified trajectories as progressive deterioration, relapsing, progressive amelioration and stable, and the detail results are presented per symptom domains as follows.

\section{Positive symptoms}

As presented in Table 1a, four studies ${ }^{19,20,57,65}$ investigated the trajectory of positive symptoms in patients with first-episode schizophrenia spectrum disorders with no or prior antipsychotics treatment for less than three months. The duration of follow-up and frequency of assessment ranged from six weeks to 10 years and five to seven times, respectively. Two studies ${ }^{19,65}$ have used the Scale for the Assessment of Positive Symptoms (SAPS) to assess positive symptoms and identified five trajectories with more than one-third of patients subtyped as decrease positive symptoms or good responders. The other two studies used the Positive and Negative Syndrome Scale (PANSS) 


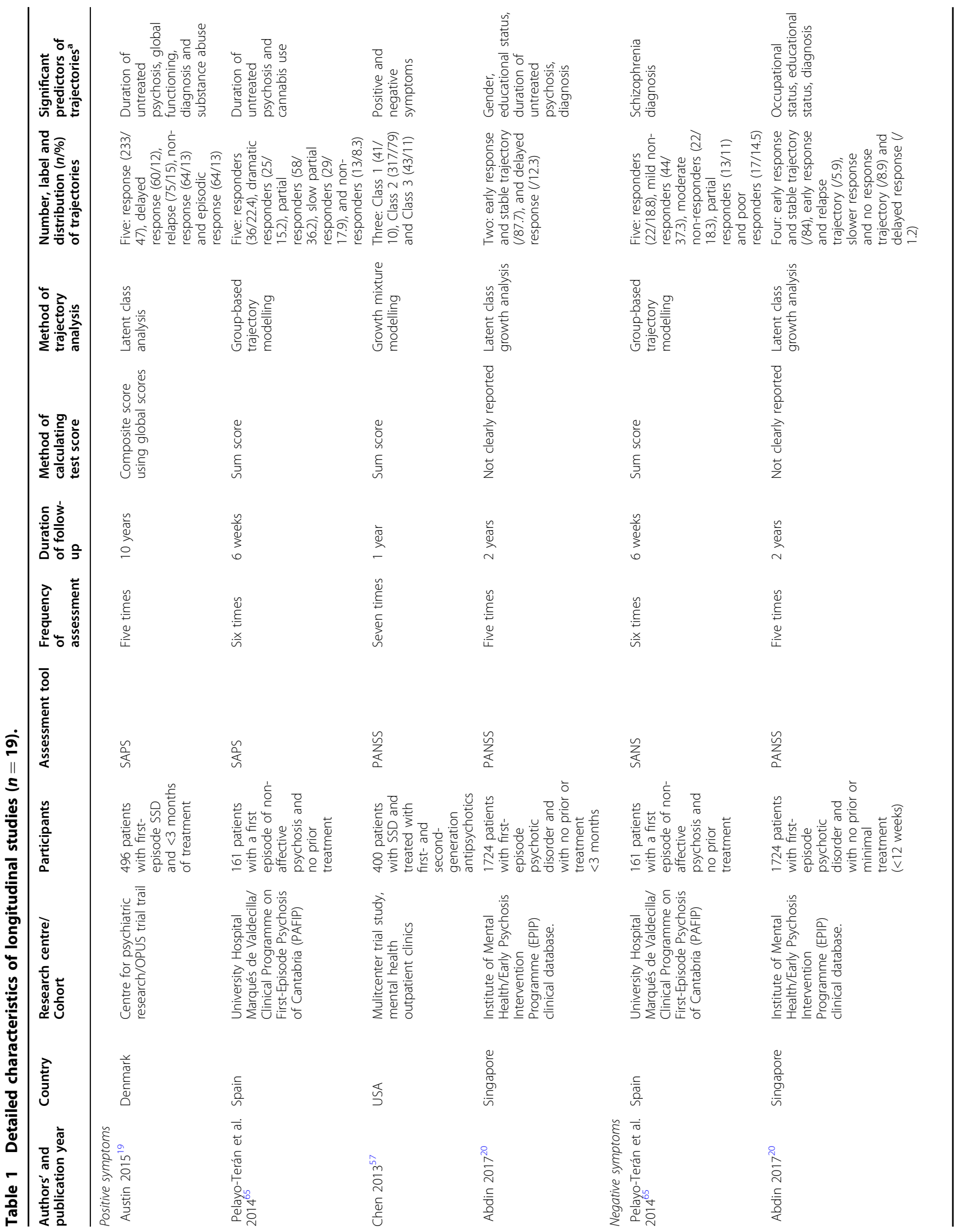




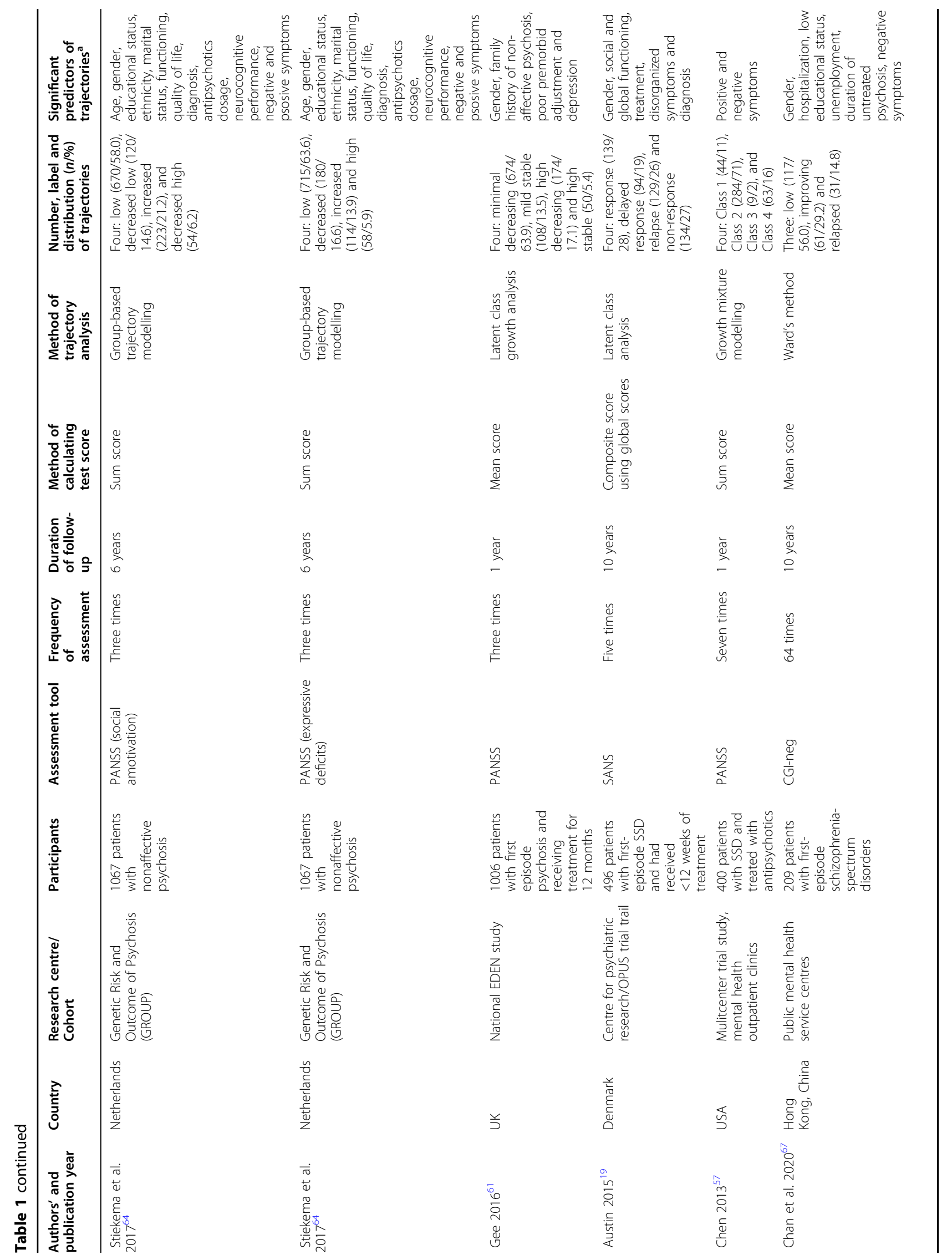




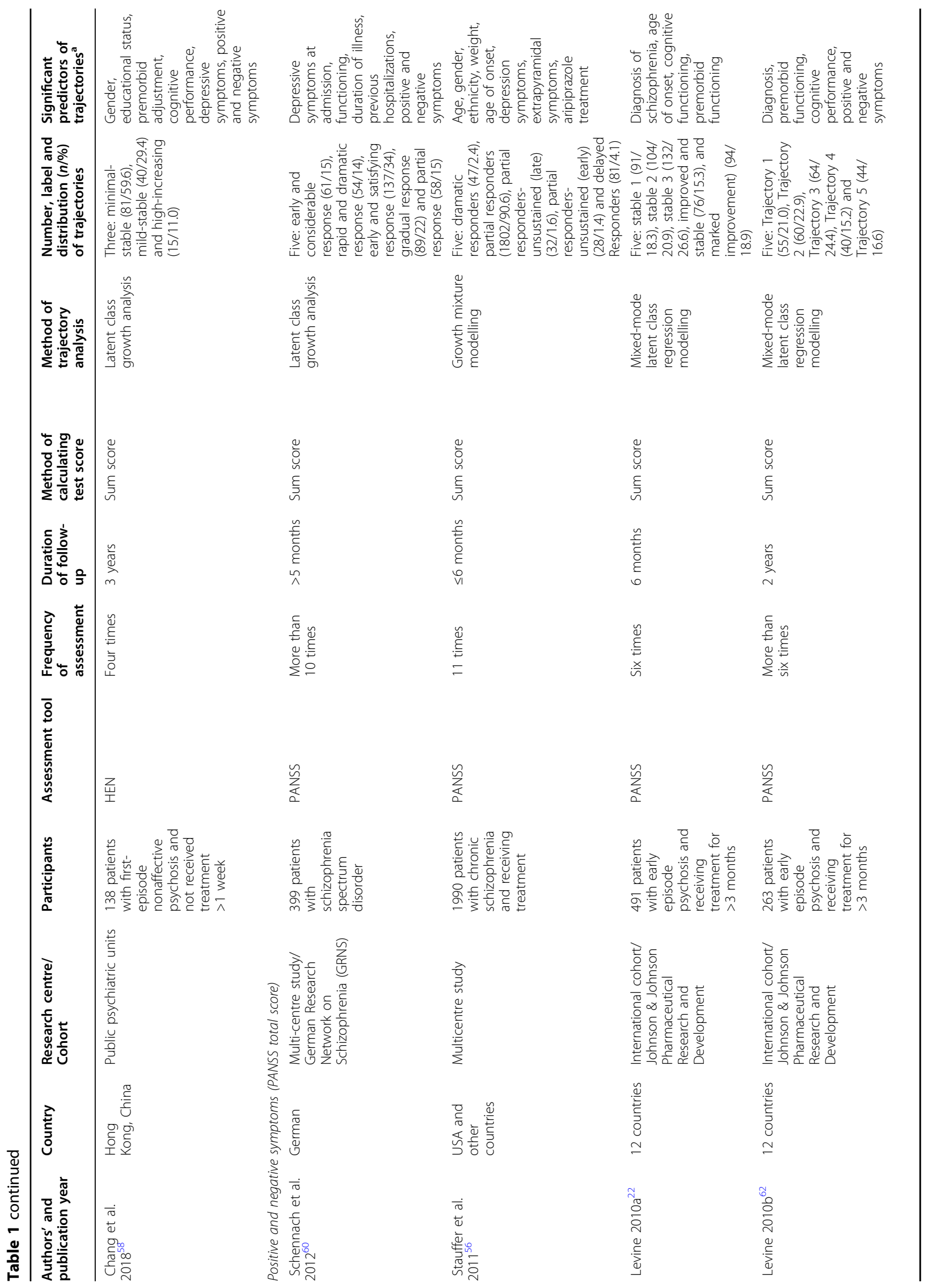




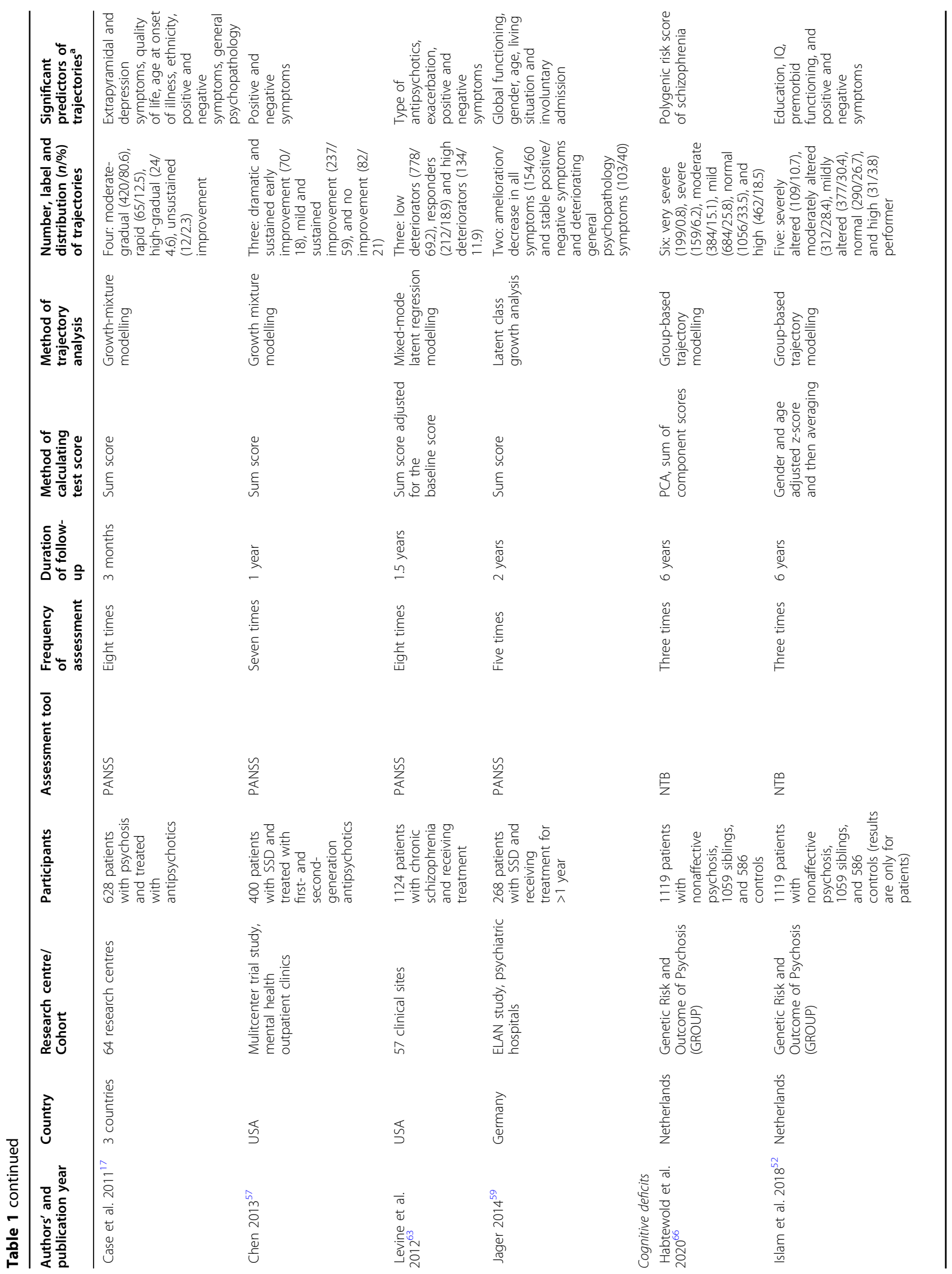




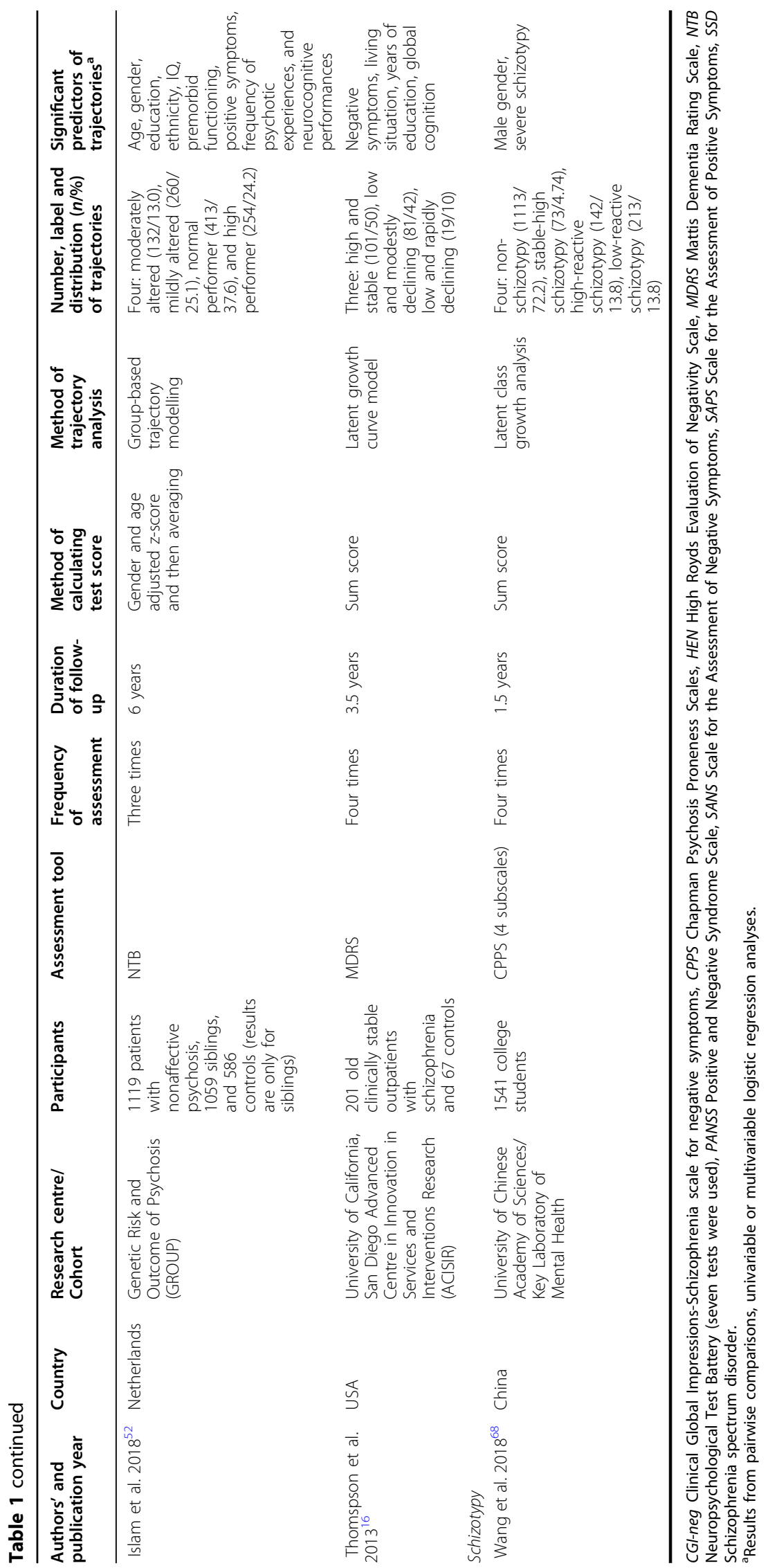


tool to assess positive symptoms and identified three trajectories that most of them grouped to class two ${ }^{57}$ and two trajectories being in the most of the cases early response and stable trajectory over time ${ }^{20}$. The identified predictors were male gender, low educational status, substance use, diagnosis with schizophrenia, long duration of untreated psychosis, poor global functioning, and severe baseline positive and negative symptoms (Fig. 2).

\section{Negative symptoms}

Eight longitudinal studies ${ }^{19,20,57,58,61,64,65,67}$ explored negative symptom trajectories among patients with first- episode non-affective psychosis with no prior or minimal treatment up to three months (Table 1b). Two studies ${ }^{19,65}$ used the Scale for the Assessment of Negative Symptoms (SANS), four studies ${ }^{20,57,61,64}$ used the PANSS scale and two studies used the High Royds Evaluation of Negativity Scale $^{58}$ and Clinical Global Impressions-Schizophrenia scale $^{67}$ to assess negative symptoms. The duration of follow-up and frequency of assessment ranged from 6 weeks to 10 years and three to 64 times, respectively. Five studies ${ }^{19,20,57,61,64}$ identified four trajectories of negative symptoms with variable patterns, whereas one study $^{65}$ found five trajectories with approximately half of them had persistent symptoms or poor response to

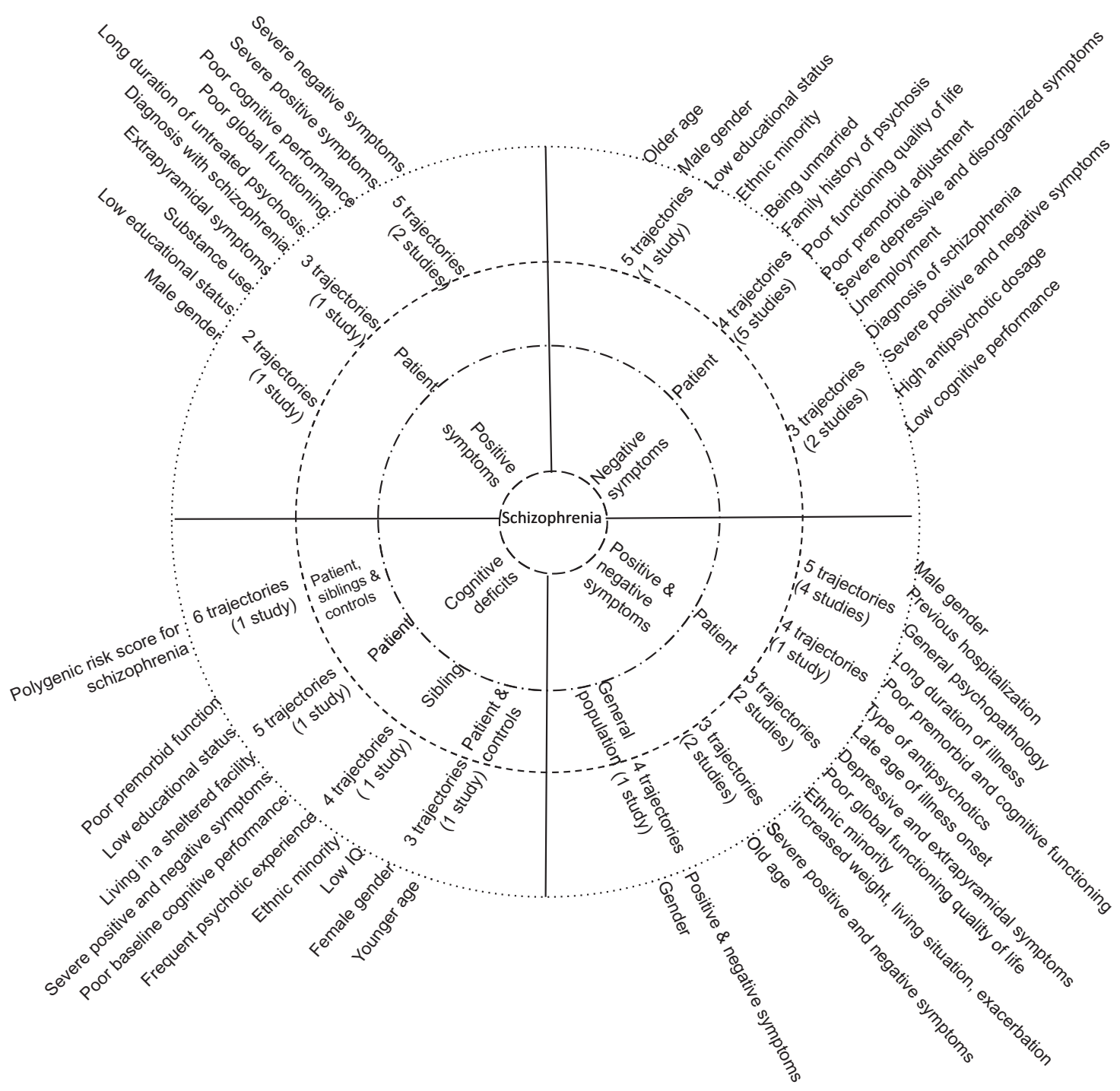

Fig. 2 Schizophrenia spectrum circle illustrating the schizophrenia symptoms and cognitive deficits (innermost circle), sample groups (inner circle), identified trajectories (outer circle) and predictors (outermost circle) in longitudinal studies. Findings are read and interpreted based on the line up in the circle. 
treatment. The other two studies ${ }^{58,67}$ found three trajectories with most of the participants had minimally stable negative symptoms. Our review depicted that trajectories of negative symptoms were predicted by older age, male gender, low educational status, ethnic minority, being unmarried, family history of psychosis, long duration of untreated psychosis, poor premorbid adjustment, severe depressive and disorganized symptoms, diagnosis of schizophrenia, unemployment, poor functioning and quality of life, high antipsychotics dosage, low cognitive performance, and high level of baseline negative and positive symptoms (Fig. 2).

\section{Positive and negative symptoms}

Combining both positive and negative symptom dimensions, which is illustrated in Table 1c, eight stu$\operatorname{dies}^{17,22,56,57,59,60,62,63}$ inspected trajectories in patients with first-episode or chronic schizophrenia with antipsychotics treatment for more than three months and all of these studies used the PANSS scale to measure positive and negative symptoms. The duration of follow-up and frequency of assessment ranged from three months to two years and five to ten times, respectively. Among these studies, four ${ }^{22,56,60,62}$ of them revealed five trajectories, two ${ }^{57,63}$ of them revealed three trajectories, one study ${ }^{17}$ found four trajectories and another study ${ }^{59}$ found two trajectories with substantial difference in the nature, pattern and distribution of trajectories. Symptom trajectories were predicted by older age, male gender, ethnic minority, increased weight, diagnosis with schizophrenia, late age of illness onset, depressive and extrapyramidal symptoms, general psychopathology, type of antipsychotics treatment (e.g., aripiprazole, olanzapine), exacerbation, long duration of illness, poor premorbid and cognitive functioning, low global functioning and quality of life, living situation, involuntary admission, previous hospitalization and severe baseline positive and negative symptoms (Fig. 2).

\section{Cognitive deficits}

As shown in Table $1 \mathrm{~d}$, three studies investigated the trajectories of global cognitive deficits in patients with first-episode psychosis patients, their siblings and healthy controls $^{52,66}$, and clinically stable outpatients with schizophrenia (SCZ) together with healthy controls ${ }^{16}$. The first six-year longitudinal study ${ }^{52}$, which cognitive function was assessed by the cognitive battery test, depicted five trajectories of cognitive impairment in patients (i.e., most of them with mild to moderate deficits) and four trajectories in healthy siblings (i.e., most of them had normal cognitive function). The second study ${ }^{66}$, which was the follow-up of the previous study, found six cognitive trajectories (i.e., nearly half of the population had mild to severe cognitive impairment) by combining patients, siblings and controls. The third longitudinal study $^{16}$ have used the Mattis Dementia Rating Scale and reported three trajectories (i.e., half of them with high and stable trajectory) of global cognitive function by combining patients and controls. Two studies found that patients with poor cognitive trajectories had younger age, low educational status, non-Caucasian ethnicity, lived in a sheltered facility, low IQ, poor premorbid adjustment, severe positive and negative symptoms, and low baseline cognitive performance ${ }^{16,52}$. Likewise, siblings with poor cognitive trajectories had younger age, female gender, low educational status, non-Caucasian ethnicity, low IQ, poor premorbid adjustment, severe schizotypy, frequent positive psychotic experience, and low baseline cognitive performance (Fig. 2) ${ }^{52}$. One study discovered that polygenic risk score for schizophrenia significantly predicted poor long-term cognitive trajectory in combined samples of patients, siblings and controls ${ }^{66}$.

\section{Schizotypy}

A single longitudinal study assessed schizotypy in healthy college students using the Chapman Psychosis Proneness Scales (CPPS) and found four trajectories, in which nearly three-fourths of students were categorized as non-schizotypal ${ }^{68}$. This study also found that male gender and a high level of baseline schizotypy significantly predicted trajectories (Table 1e, Fig. 2).

In summary, when we inspecting the longitudinal study's findings shown in Table 1, studies that found the same number of trajectories were substantially different concerning participants composition (patient, sibling and controls), assessment instruments, symptom dimensions, frequency of assessment, duration of follow-up, methods used to generate a composite score, data-driven methods applied, label, proportion, pattern and type of trajectories, and identified predictors. In addition, there was no link between the numbers and types of trajectories and the use of trajectory analysis methods, study population and symptom dimensions.

\section{Cross-sectional studies}

Of the 53 included studies, 34 studies were crosssectional (Table 2) that conducted in different groups of population. The total sample size per study ranged from 62 to 8231 individuals irrespective of participants' diagnostic status. The reported clustering methods were Kmeans or non-hierarchical clustering analysis ${ }^{21,53,55,69-76}$, Ward's method or hierarchical analysis ${ }^{77-83}$, K-means clustering and Ward's method ${ }^{18,38,54,84-89}$, latent class or profile analysis ${ }^{15,90,91}$ and two-step cluster analysis ${ }^{92-94}$. One study ${ }^{95}$ identified clusters using a combination of clinical/empirical and statistical clustering methods. The model selection criteria or similarity metrics were visual inspections of the dendrogram, Pearson correlation, 


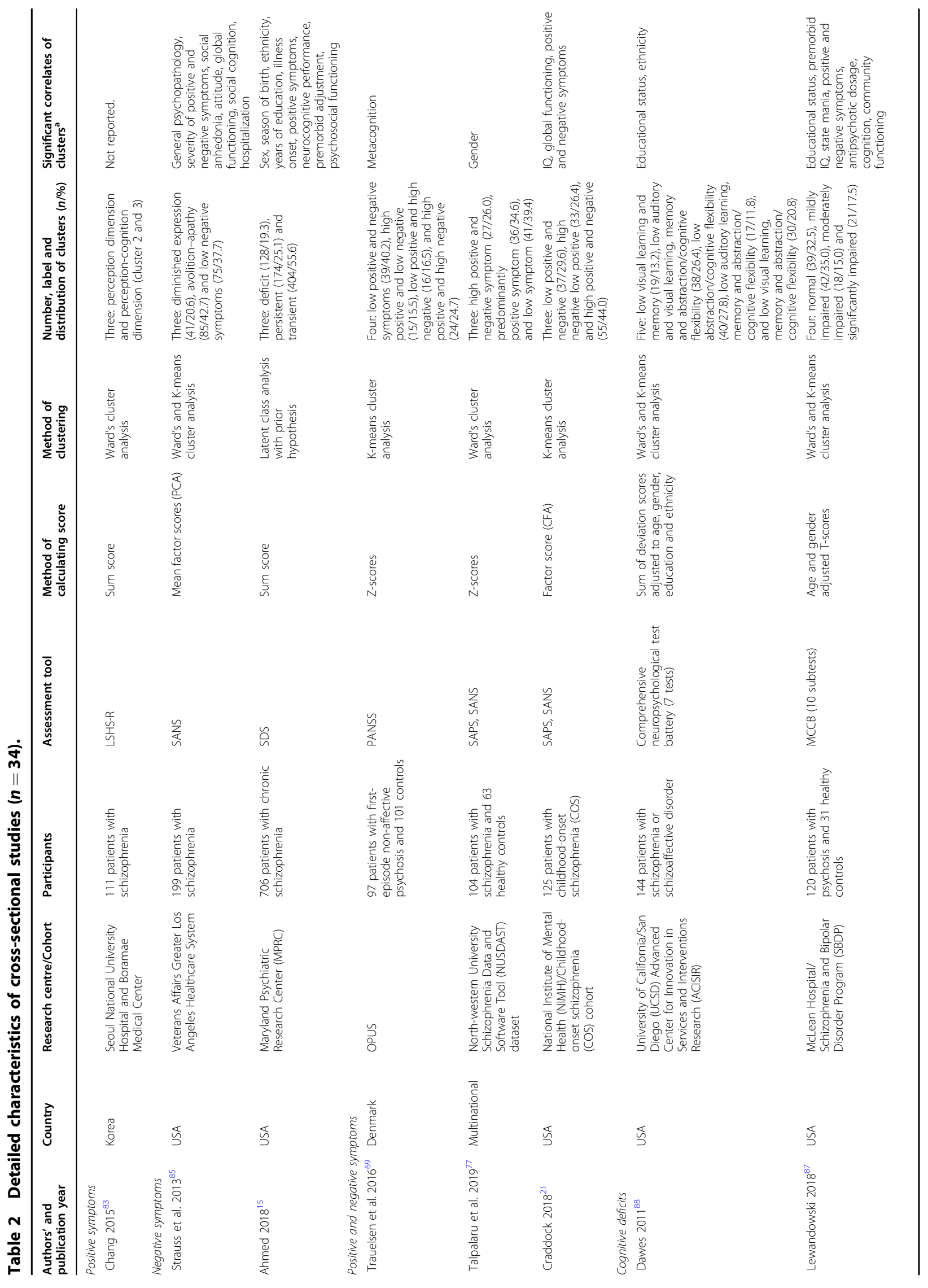




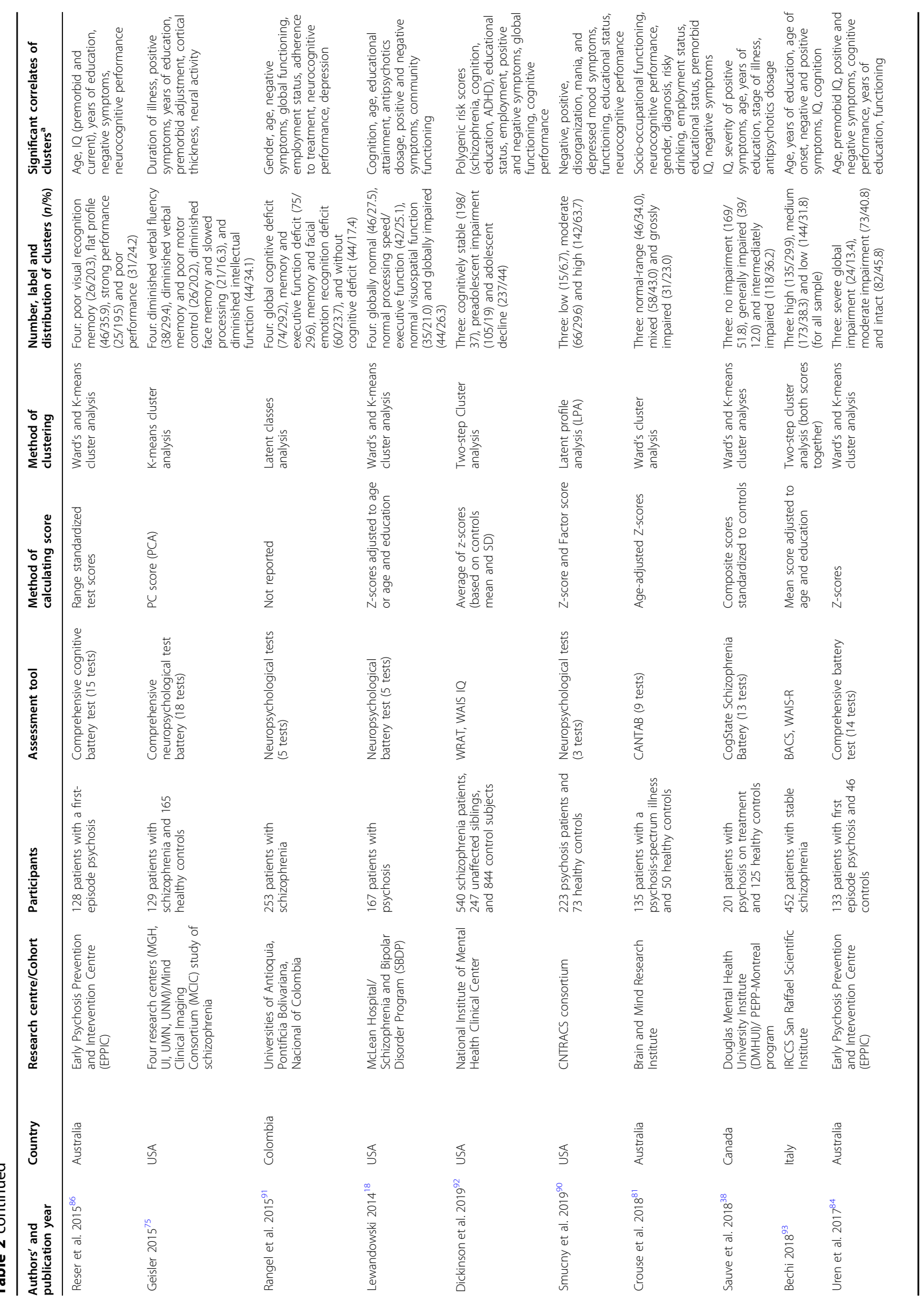




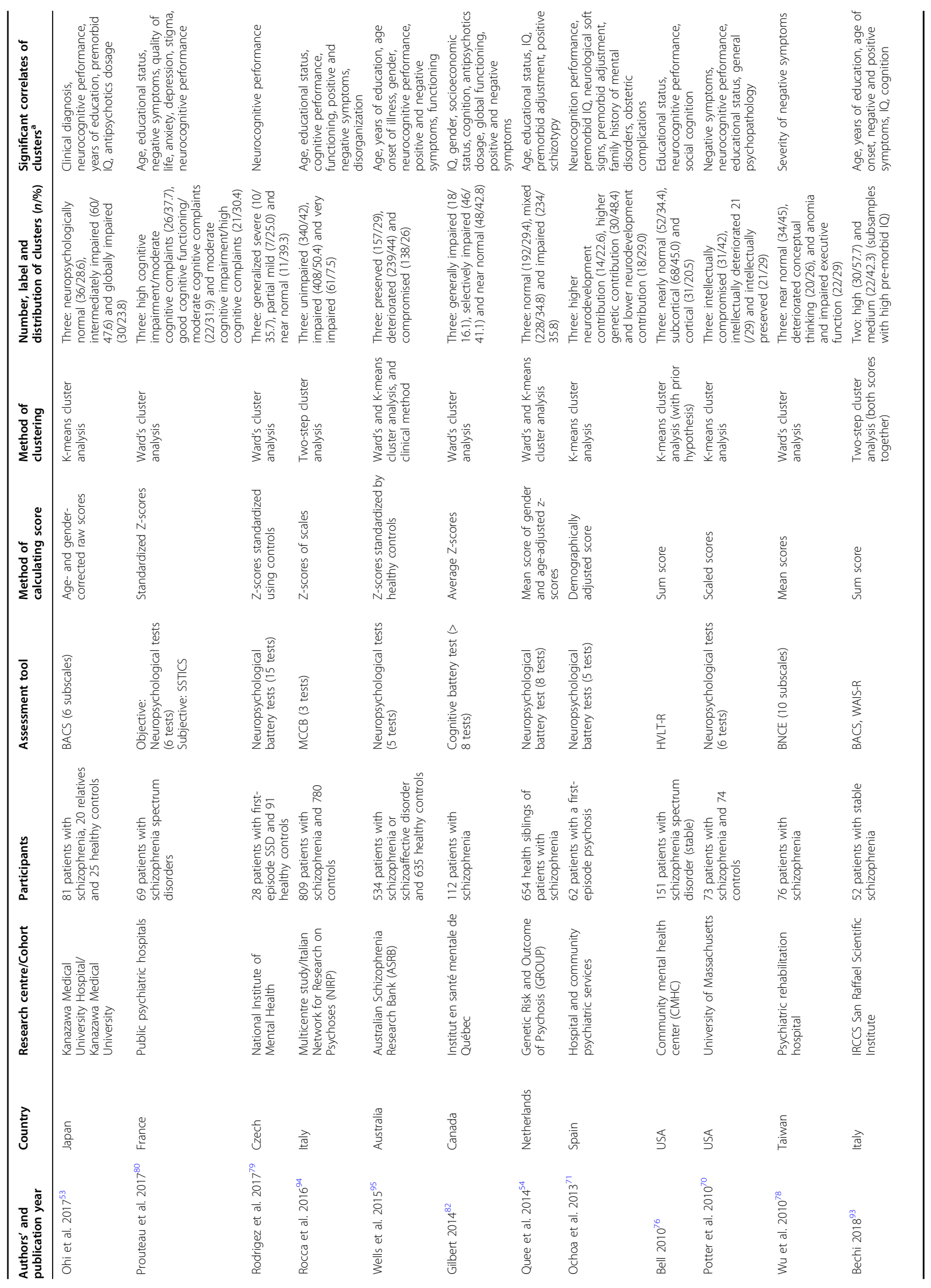




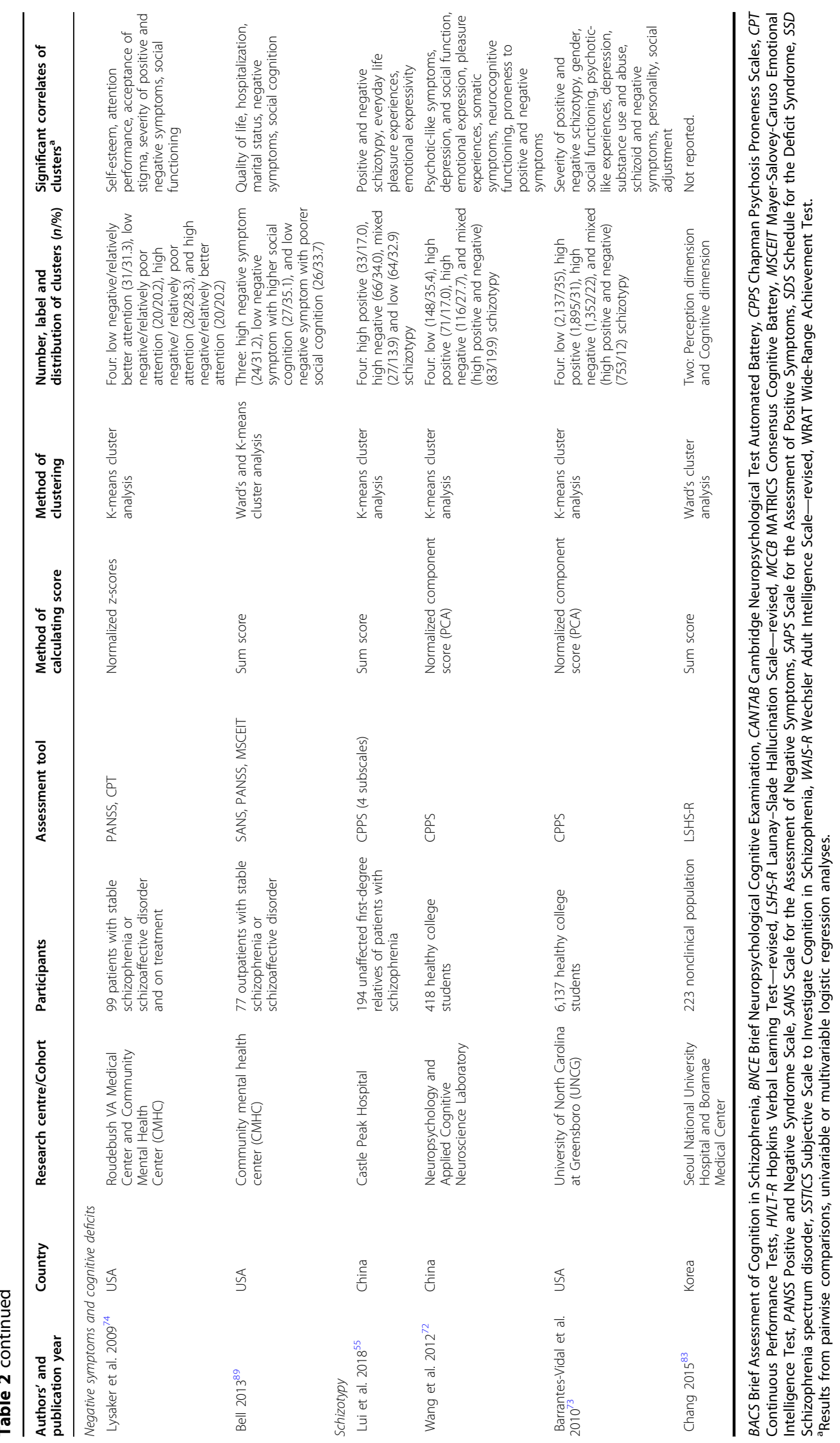


squared Euclidean distance (i.e., the most common index), agglomeration coefficients, Dunn index, Silhouette width, Duda and Hart index, elbow test, variance explained, inverse scree plot, average proportion of non-overlap, AIC, BIC, aBIC, Schwarz's BIC, Lo-Mendell-Rubin (LMR) test, adjusted LMR and BLRT.

Among the 34 studies (Table 2), 22 studies $^{18,38,53,54,70,71,75,76,78-82,84,86-88,90-95}$ reported cognitive clusters in patients with first-episode, stable or chronic schizophrenia with or without antipsychotics treatment and one study ${ }^{54}$ reported cognitive clusters in unaffected siblings. Other studies investigated trajectories of negative symptoms ${ }^{15,85}$, positive symptoms ${ }^{83}$, positive and negative symptoms $s^{21,69,77}$ in patients and positive and negative schizotypy in a nonclinical population ${ }^{55,72,73,83}$. Furthermore, two studies ${ }^{75,90}$ investigate the data-driven clusters by combining cognitive deficit and negative symptoms. Details on clusters and correlates of clusters presented per symptom dimensions as follows.

\section{Positive symptoms}

Only one study ${ }^{83}$ assessed hallucinatory experience in patients with schizophrenia using Launay-Slade Hallucination Scale-Revised (LSHS-R) and identified three clusters (Table $2 \mathrm{a})^{83}$. Given this was an explanatory study, correlates of clusters were not studied.

\section{Negative symptoms}

Two studies ${ }^{15,85}$ reported three clusters of patients with (chronic)schizophrenia based on the negative symptoms that assessed by the SANS scale ${ }^{85}$ and Schedule for the Deficit Syndrome (Table $2 b)^{15}$. Identified clusters were significantly correlated with male gender, ethnic minority, low educational status, summer season of birth, early age onset of illness, severity of positive and negative symptoms, poor cognitive performance, poor functioning, high level of general psychopathology, previous hospitalization, poor premorbid adjustment, social anhedonia and poor attitude (Fig. 3).

\section{Positive and negative symptoms}

Two studies ${ }^{21,77}$ assessed positive and negative symptoms in patients with childhood-onset or first-episode schizophrenia using the SAPS and SANS scales, respectively and found three clusters, while another study ${ }^{69}$ used the PANSS scale and found four clusters (Table 2c). Reported symptom clusters were characterized as low positive and negative symptoms, high positive and low negative, low positive and high negative, and high positive and high negative though the patterns and distributions of clusters were different across studies. Identified clusters were significantly correlated with male gender, low IQ, poor global functioning, poorer metacognitive ability, and high level of positive and negative symptoms (Fig. 3).

\section{Cognitive deficits}

Of the 22 studies conducted on neurocognitive deficits, 17 studies $^{38,53,70,71,76,78-82,84,90,92-95}$ found three clusters, five studies ${ }^{18,75,86,87,91}$ reported four clusters and one study ${ }^{88}$ discovered five clusters among patients (Table 2d). Most studies assessed global cognitive function using a comprehensive neuropsychological test that included three to 18 cognitive subtests. Poor cognitive function in patients was associated with age, gender, non-Caucasian ethnicity, low socioeconomic and educational status, poor premorbid adjustment, low premorbid and current IQ, early age of illness onset, long duration of illness, severe positive and negative symptoms, poor social cognition, high antipsychotics dosage, use of second-generation antipsychotics, and poor functioning and poor quality of life (Fig. 3). In siblings, one study ${ }^{54}$ found three cognitive clusters in unaffected siblings that associated with young age, low educational status, low IQ, poor premorbid adjustment and severe positive schizotypy (Table 2d, Fig. 3$)^{54}$. One study ${ }^{92}$ found that polygenic score (PRS) for schizophrenia, cognition, educational attainment and attention deficit hyperactivity disorder (ADHD) correlated with cognitive clusters in patients and their unaffected siblings.

\section{Negative symptoms and cognitive deficits}

One study ${ }^{89}$ found three clusters of (out)patients with stable schizophrenia spectrum disorder by combining social cognition that assessed by the Mayer-SaloveyCaruso Emotional Intelligence Test and negative symptoms that assessed by the PANSS scale, whereas another study $^{74}$ found four clusters in patients by combining neurocognition that assessed by Continuous Performance Tests and negative symptom that assessed by the PANSS scale (Table 2e). Clusters were significantly correlated with being unmarried, poor self-esteem, low cognitive (attention, social) performance, stigma, severity of positive and negative symptoms, poor social functioning and quality of life, and previous hospitalization (Fig. 3).

\section{Schizotypy}

Three studies investigated schizotypy in unaffected firstdegree relatives of patients with schizophrenia ${ }^{55}$ and healthy college students ${ }^{72,73}$ using the CPPS scale and found four clusters, whereas another study ${ }^{83}$ found two clusters based on hallucinatory experience that assessed by LSHS-R scale in healthy general population (Table $2 \mathrm{f}$ ). Schizotypy clusters were significantly associated with male gender, lack of pleasure experiences, difficulty of emotional expression, psychotic-like symptoms, severity of positive and negative schizotypy, depressive, schizoid and somatic symptoms, poor social and cognitive functioning, substance abuse and poor personality (Fig. 3). 


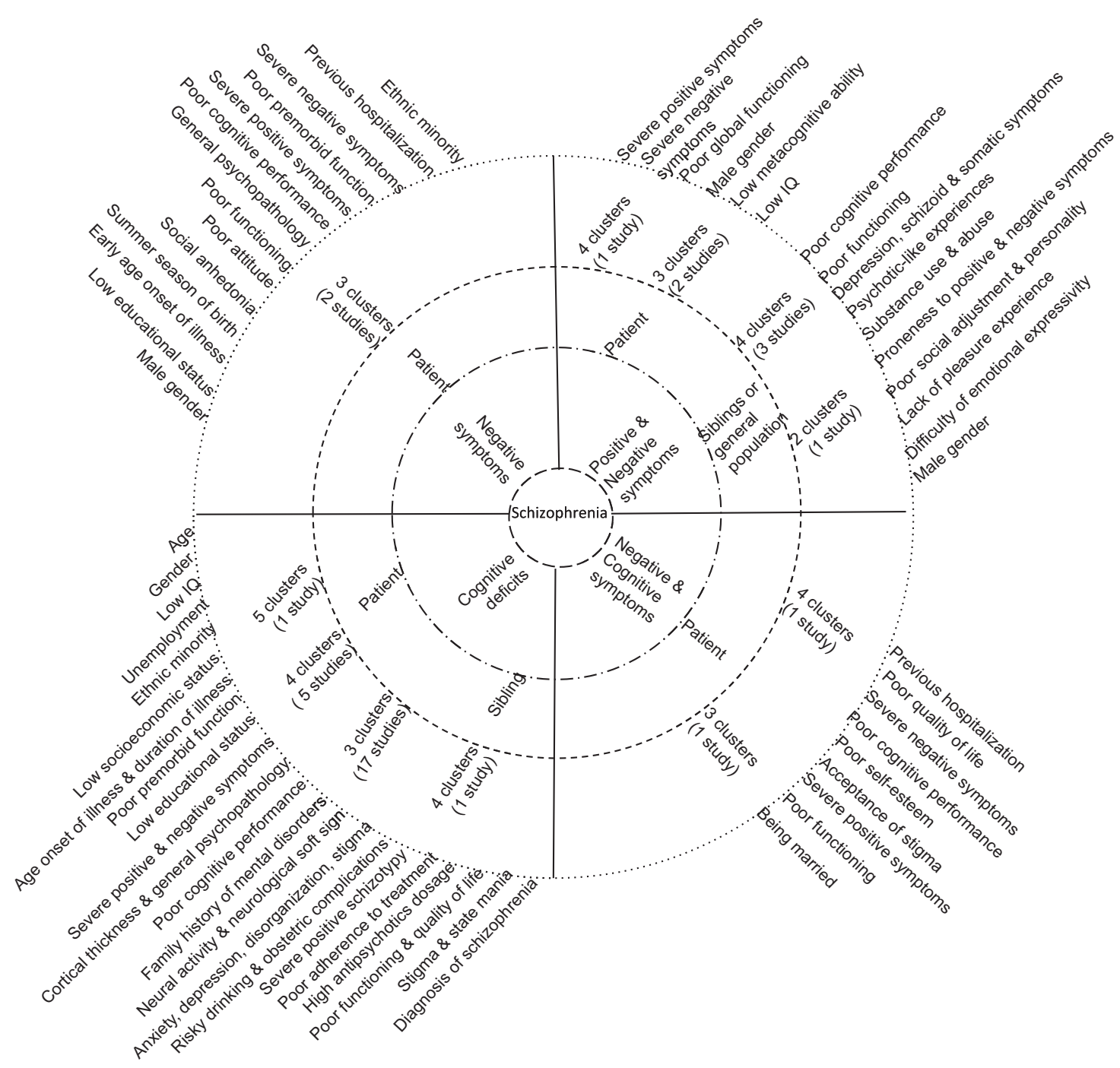

Fig. 3 Schizophrenia spectrum circle illustrating the schizophrenia symptoms and cognitive deficits (innermost circle), sample groups (inner circle), identified clusters (outer circle) and correlates (outermost circle) in cross-sectional studies. Findings are read and interpreted based on the line up in the circle.

To summarize, as we observed in longitudinal studies, cross-sectional studies that found the same number of clusters were conducted in a different group of samples and used various assessment instruments and methods of generating composite scores and clustering. The labeling, pattern, proportion, and type of clusters were remarkably different. Generally, three clusters were the most replicated number of clusters and characterized by low (severe deficits), mixed (intermediate deficits) and high (intact or normal performance) cognitive function. In addition, cognitive clustering, such as verbal fluency deficit, verbal memory and executive function deficit, face memory and processing deficits, or global cognitive deficits were revealed. Cross-sectional studies that found the same number of clusters were largely different in the characteristics of study population, pattern of identified clusters, symptom dimensions, methodology of assessment, applied data-driven methods and identified associated factors.

Overall, as shown in Table 3, the reviewed studies reported two to six clusters or trajectories and 58 factors that linked with identified clusters and/or trajectories across all study participants and symptom dimensions. The most common associated factors were old age, male gender, non-Caucasian ethnicity, low educational status, late age of illness onset, diagnosis of schizophrenia, several general psychopathology and depressive symptoms, severe positive and negative symptoms, low cognitive performance, and poor premorbid functioning, quality of life and global functioning. 
Table 3 Heatmap summary of clusters/trajectories and predictors across study participants, symptom dimensions and study design.

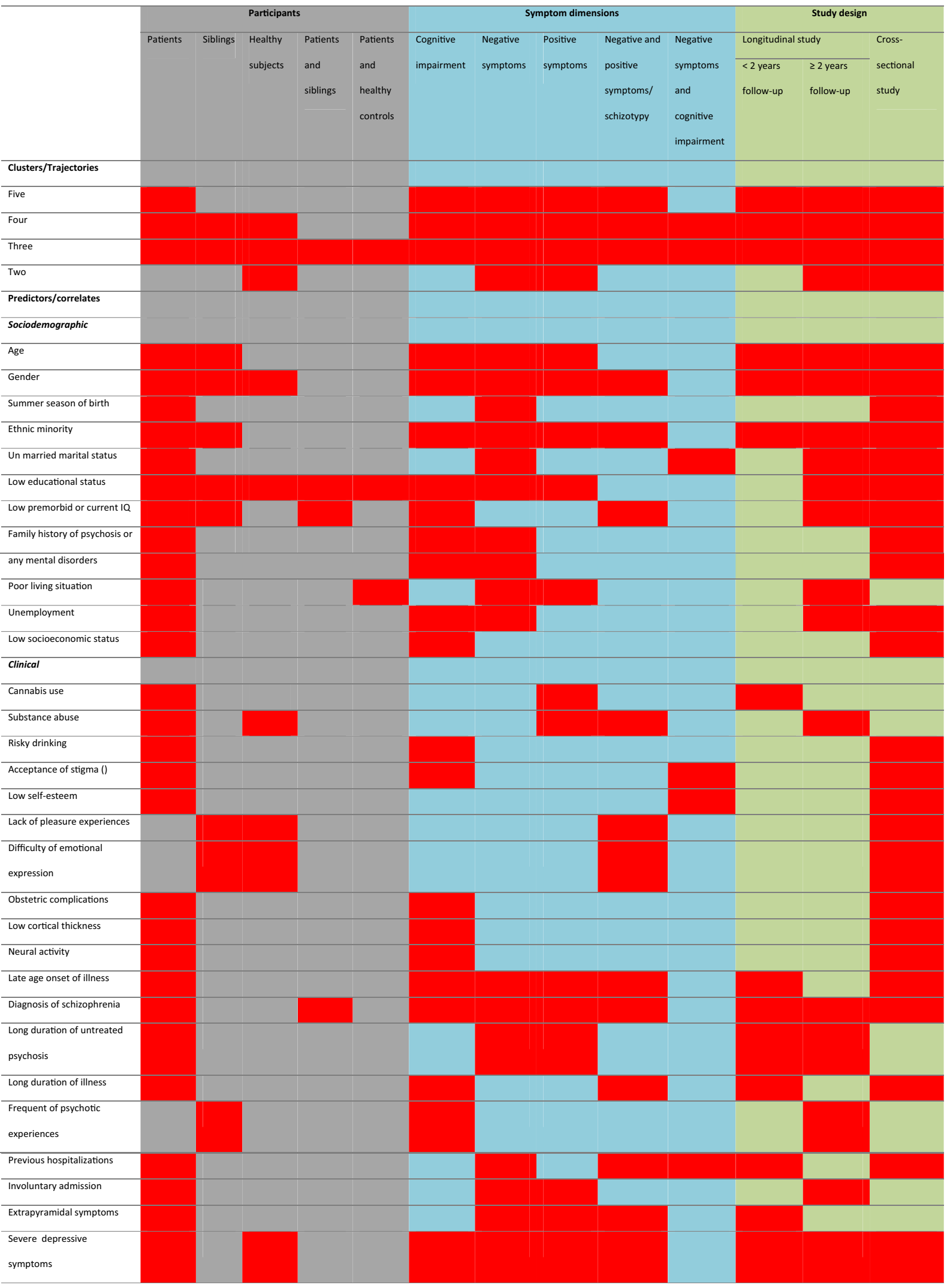




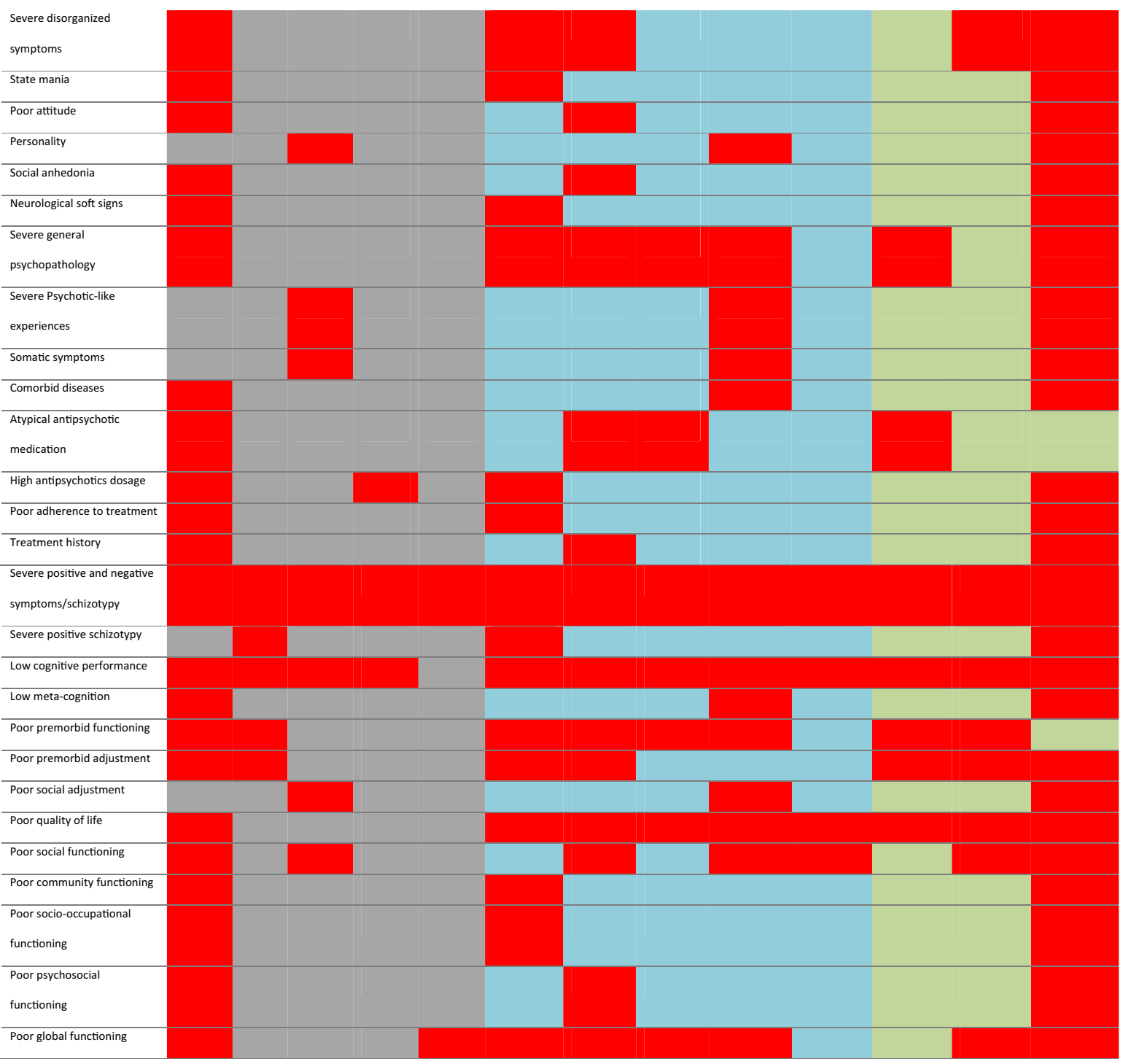

This table/map can only be read and interpreted horizontally. For example, five clusters/trajectories were found in both longitudinal and cross-sectional studies among patients based on schizophrenia symptoms and cognitive deficits [all red boxes]. The same procedure applies to predictors. For example, age found to be the predictor of clusters/trajectories of schizophrenia symptoms and cognitive deficits in longitudinal and cross-sectional studies among patients and siblings [all red boxes].

\section{Discussion}

To our knowledge, this is the first comprehensive systematic review based on recent cross-sectional and longitudinal data-driven studies in positive and negative symptoms, and cognitive deficits in patients with schizophrenia spectrum disorders, their relatives and healthy people. Our review has three major findings. First, longitudinal trajectory-based studies found two to five positive and negative symptoms trajectories in patients and four to six cognitive trajectories in patients, siblings, controls, or combined samples. Second, cross-sectional cluster-based studies identified three positive and negative symptoms clusters among patients and four positive and negative schizotypy clusters among healthy siblings. Additionally, three to five cognitive clusters were discovered in patients and their unaffected relatives. Third, numerous sociodemographic, clinical and genetic factors that determine trajectories and/or clusters were identified.

We showed that longitudinal and cross-sectional studies in patients, their siblings and healthy general population have inconsistently identified two to five trajectories/ clusters and various predictors across the schizophrenia symptoms and cognitive deficits. Several shortcomings across studies may cause this inconsistency. Previous longitudinal studies did not uniformly research symptoms and cognitive deficits. For example, only three 
studies ${ }^{16,52,66}$ longitudinally investigated cognitive trajectories, but 22 cross-sectional studies investigated cognitive clusters. Utterly, none of the reviewed longitudinal and cross-sectional studies also validated their model using empirical methods or comparable statistical methods though they have used different complex data-driven methods. Accumulating evidence showed that the number of classes in the optimal model derived from one method can be remarkably different compared to the other method ${ }^{96}$. Given that these studies were conducted in patients at a different stage at diagnosis, disease course or severity of illness and treatment status, the results may not be expectedly consistent as well. For example, studies that included only first-episode psychosis, chronic or stable patients may identify smaller clusters than studies that included a mixture of patients with first-episode and chronic psychosis or patients with severe illness. Additionally, since the reported studies were conducted in more than 20 countries, the use of different treatment strategies and assessment methods in different countries could further confound the assessment of symptoms and clinical heterogeneity. Obviously, in patients who are treated, the observed symptoms and cognitive characteristics are the product of those features that were present before treatment and the response to treatment. Moreover, the different measurement tools may lead to discrepant results. For instance, the discrepancy of negative and positive symptoms trajectories (or cross-sectional clusters) might partly be attributable to the use of a specific negative (e.g. SANS) and positive (e.g. SAPS) symptom scale or a more general symptom scale (e.g. PANSS) that included items measuring cognitive or disorganization symptoms. Additionally, some studies administered up to 18 different neuropsychological tests to measure cognition while others have used as few as two or three cognitive assessment tests.

We further observed common methodological limitations across studies. Firstly, the reviewed studies included various groups of participants from different age groups and ethnicities. Secondly, while the comparison of patient clusters and trajectories with healthy siblings or controls could provide an accurate means of disentangling the heterogeneity and causes of heterogeneity of schizophrenia symptoms, only four studies (three were crosssectional studies) examined clusters in siblings. Likewise, most studies used healthy controls to standardize patients neurocognitive composite scores, and a few other studies used controls to compare the distribution of patient clusters or trajectory groups. Thirdly, substantial differences between studies were also noted in constructing composite scores, use of model selection criteria and method of parameter estimation. Fourthly, we observed several ways of subtyping and nomenclature for clusters or trajectories, which may be difficult for clinicians to translate the evidence in diagnosing and treating diseases. This is due to the lack of a standard for designing a study (e.g. adequate sample size), reporting data analysis approaches and publishing results ${ }^{42}$.

Generally, we saw that studies conducted in patients with similar stages of illness (i.e, first-episode, stable, chronic stage or with or without treatment) and used similar assessment methods (i.e., SANS, SAPS or PANSS) showed some level of similarity in results with respect to identified trajectories and predictors, but studies are largely different in duration of follow-up, frequency of assessment and methods used to assess symptoms or cognition. By the same token, studies that used the similar data-driven statistical methods showed similarity in the number of identified trajectories/clusters, but largely different in study population, stage of illness, use of measurement tool, duration of follow-up, frequency of assessment and identified factors. Moreover, studies with duration of follow-up less than two years and above two years showed a similar level of heterogeneity in symptoms and cognitive deficits and identified predictors. In addition, a 10-year study with five times assessment showed similar findings with a 6 week study with every week assessment on positive symptoms. On the other hand, a 2year study with five times assessment identified only two trajectories. Despite these facts, all studies interestingly showed heterogeneity of symptoms and cognitive deficits at various level with "four trajectories" is the most replicated in longitudinal studies and "three clusters" is the most replicated in cross-sectional studies. Besides, these studies consistently reported age, gender, ethnicity, educational status, age of illness onset, diagnosis, general psychopathology and depressive symptoms, positive and negative symptoms, cognitive performance, functioning and quality of life as determinant factors of trajectories and/or clusters.

In the era of team science and big data, the use of datadriven statistical methods is becoming increasingly popular for the analysis of longitudinal repeated measures (i.e., latent growth mixture models (LGMMs)) and crosssectional (i.e., cluster analysis) data (Fig. 4). In our review, we observed that LGMMs, such as GMM, latent class growth analysis (LCGA), mixed mode latent class regression modelling and group-based trajectory modelling (GBTM) were commonly used data-driven methods in longitudinal studies. LGMMs can identify realistic categories based on temporal patterns of change in outcome by assuming the existence of latent classes or subgroups of subjects exhibiting similarity with regard to unobserved (latent) variables ${ }^{19,97}$. LGMMs have four advantages for modelling longitudinal data. First, they are flexible and data-driven methods that can accurately reveal actual heterogeneity. Second, they allow the classification of individual subjects into latent classes based 


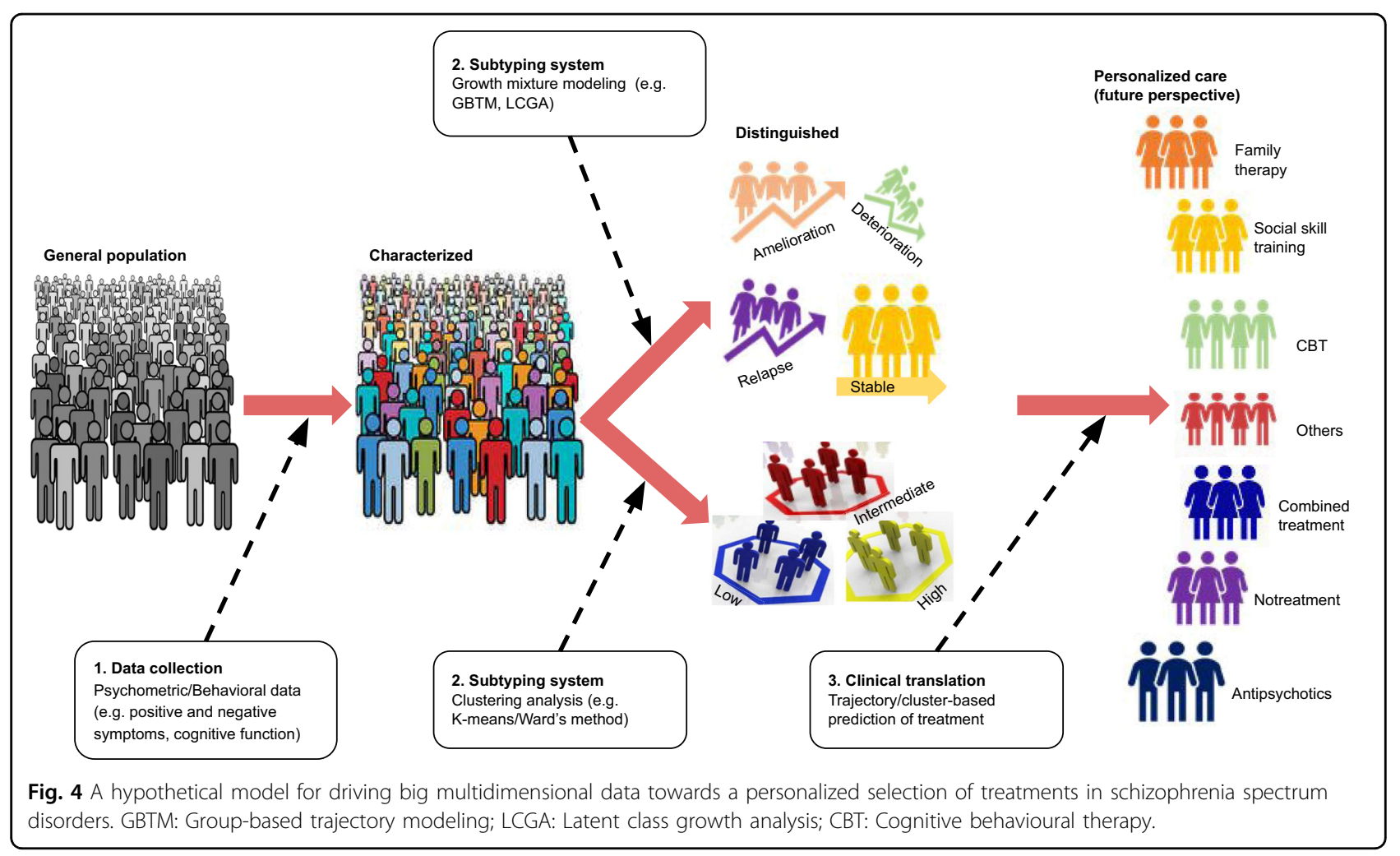

on the largest probability of class membership. Third, they are sensitive to the pattern of change over time and robust in the presence of missing data. Fourth, subject-level predictors can be directly assessed for association with class membership and hence with different trajectory subtypes ${ }^{16,19,97}$. Cluster analysis, which is commonly used in cross-sectional studies, is also a data-driven approach for classifying people into homogeneous groups by determining clusters of participants that display less within-cluster variation relative to the between-cluster variation $^{81}$. Among the reviewed cross-sectional studies, K-means and Ward's method clustering analyses were commonly used alone or in combination. K-means cluster analysis is a non-hierarchical form of cluster analysis appropriate when previous evidence or hypotheses exist regarding the number of clusters in a sample ${ }^{74}$. On the other hand, Ward's method is a hierarchical cluster analysis aiming to determine group assignment without prior hypothesis ${ }^{74}$. It is believed, $\mathrm{K}$-means cluster analysis can handle larger data sets compared with Ward's method ${ }^{73}$.

The results of statistical subtyping approaches, such as cluster or trajectory analysis depend on mathematical assumptions, type of data, number of variables or tests, sample size and sampling characteristics. Therefore, the models can be unstable and parameter estimates of clinical symptoms may not converge to a consistent set of subgroups and lack a direct relationship to clinical reality ${ }^{59,87,98}$. For example, intermediate clusters and trajectories substantially vary between studies that used the same cluster or trajectory analysis method ${ }^{87}$. We advocate that study results from data-driven methods should be applicable, comparable, generalizable and interpretable into clinical practice. As a result, we recommend to validate models using at least one additional comparable statistical methods, combine statistical methods of subtyping with empirical/clinical methods, or work together with clinicians to create a common understanding and clinically relevant clustering or trajectories nomenclatures. Furthermore, it is relevant to replicate clusters or trajectory groups using independent samples, different assessment tools that measure the same construct, or different linkage methods ${ }^{38,99}$. Finally, further studies are required that focus on longitudinal study design, unaffected siblings, genetic markers and more detailed measures of brain network function for improving our understanding of the biological mechanism underlying heterogeneity of schizophrenia.

Future clinical advances may benefit from the subgrouping of patients to implement tailored therapy. In our review, we observed that several longitudinal studies were conducted based on drug response. One study found individuals who treated with aripiprazole had delayed response ${ }^{56}$, whereas another study found olanzapine treated patients had good response ${ }^{63}$. Another study also 
revealed individuals receiving standard treatment, compared to assertive treatment, showed delayed negative symptom trajectory ${ }^{19}$. Furthermore, individuals with substantial cognitive deficit received high dose of antipsychotics ${ }^{18,82,87}$. Subtyping of symptoms and cognitive deficits can also contribute to uncover the biological basis of individual symptoms, rather than studying constellation of co-occurring symptoms ${ }^{1}$. The identified factors associated with clusters and/or trajectories could be used for developing a clinical risk prediction model for highrisk individuals with prodromal symptoms ${ }^{100,101}$.

Thus far, findings from this review showed that datadriven approaches could have substantial role to optimize the efficacy of personalized care by predicting individual susceptibility to disease, providing accurate assessments of disease course, contribute to best-choice of early intervention, and selecting treatments (e.g., antipsychotics, cognitive behavioral therapy, social skill training, family therapy) targeting subgroups of patients with similar phenotypic or psychosocial characteristics (Fig. 4) ${ }^{102}$. When data-driven methods are implemented on samples/cohorts following different pharmacological and non-pharmacological interventions, then, we believe that our proposed model (Fig. 4) can identify individuals who successfully treated, not treated or even harmed and who needs further intervention and close follow-up to protect from unnecessary cost and side effect of medication(s). Therefore, findings from our review could assist in the implementation of personalized and preventive strategies for clinical practice at least in national or regional level.

\section{Conclusions}

Our review indicated a significant heterogeneity in results and conclusions obtained from both crosssectional and longitudinal studies in terms of the number of group membership for positive and negative symptoms and cognition as well as factors (predictors) associated with the group membership. This review also identified several methodological issues contributing to the discrepant results. Generally, the longitudinal studies identified trajectories characterized by progressive deterioration, relapsing, progressive amelioration and stability, whereas low, mixed (intermediate) and high psychotic symptoms and cognitive clusters were identified by crosssectional studies. Future studies can be more benefited from data-driven methods if applied based on pharmacological and non-pharmacological treatment responses. The use of empirical methods to distinguish more homogeneous subgroups of patients along heterogeneous symptom dimensions has gained traction in the last several years and it is an essential step toward implementation of a more precise prediction of disease risk and individualized selection of interventions.
Data availability

All relevant data were included in the paper.

\section{Acknowledgements}

We would like to forward our special gratitude to Sjoukje van der Werf, who is a medical information specialist at the University of Groningen, the Netherlands, for her support to develop the search strings and guiding the overall literature retrieval process. Tesfa Dejenie was supported by the Scholarship of University of Groningen, Groningen, the Netherlands. L.H. Rodijk was supported by the Junior Scientific Master Class of the University of Groningen, Groningen, the Netherlands.

\section{Author details}

${ }^{1}$ Department of Epidemiology, University Medical Center Groningen, University of Groningen, Groningen, The Netherlands. ${ }^{2}$ Department of Psychiatry, Rob Giel Research Center, University Medical Center Groningen, University Center for Psychiatry, University of Groningen, Groningen, The Netherlands. ${ }^{3}$ Department of Pediatric Surgery, University Medical Center Groningen, University of Groningen, Groningen, The Netherlands. ${ }^{4}$ Department of Neuroscience, University Medical Center Groningen, University of Groningen, Groningen, The Netherlands

\section{Author contributions}

T.D.H. and B.Z.A. conceived and designed the study. T.D.H. and L.H.R. did study selection and data extraction. R.B., B.Z.A. and H.M.B. led the primary work during systematic review and narrative synthesis. T.D.H. and B.Z.A. drafted the paper. L.H.R., G.S. and E.J.L. provide intellectual comments during the revision process of the paper. All co-authors contributed to the review and synthesis of the findings, and reviewed and approved the final paper.

\section{Conflict of interest}

The authors declare that they have no conflict of interest.

\section{Publisher's note}

Springer Nature remains neutral with regard to jurisdictional claims in published maps and institutional affiliations.

Supplementary Information accompanies this paper at (https://doi.org/ 10.1038/s41398-020-00919-x).

Received: 6 March 2020 Revised: 24 June 2020 Accepted: 3 July 2020 Published online: 21 July 2020

\section{References}

1. Ozomaro, U., Wahlestedt, C. \& Nemeroff, C. B. Personalized medicine in psychiatry: problems and promises. BMC Med. 11, 132 (2013).

2. Jablensky, A. The diagnostic concept of schizophrenia: its history, evolution, and future prospects. Dialogues Clin. Neurosci. 12, 271-287 (2010).

3. Owen, M. J., Sawa, A. \& Mortensen, P. B. Schizophrenia. Lancet 388, 86-97 (2016).

4. Gejman, P. V., Sanders, A. R. \& Duan, J. The role of genetics in the etiology of schizophrenia. Psychiatr. Clin. North Am. 33, 35-66 (2010).

5. Pardiñas, A. F. et al. Common schizophrenia alleles are enriched in mutationintolerant genes and in regions under strong background selection. Nat. Genet. 50, 381 (2018).

6. American Psychiatric Association. Diagnostic and statistical manual of mental disorders: DSM-5. (American Psychiatric Association, 2013).

7. Palmer, B. W., Dawes, S. E. \& Heaton, R. K. What do we know about neuropsychological aspects of schizophrenia? Neuropsychol. Rev. 19, 365-384 (2009).

8. Petrova, N. \& Dorofeikova, M. Cognition in schizophrenia: Selective impairment and factors that influence it. Eur. Psychiatry 41, S193 (2017).

9. Shmukler, A. B., Gurovich, I. Y., Agius, M. \& Zaytseva, Y. Long-term trajectories of cognitive deficits in schizophrenia: a critical overview. Eur. Psychiatry 30, 1002-1010 (2015).

10. Krukow, P. et al. Processing speed is associated with differences in IQ and cognitive profiles between patients with schizophrenia and their healthy siblings. Nord J. Psychiatry 71, 33-41 (2017). 
11. Walker, A. E., Spring, J. D. \& Travis, M. J. Addressing cognitive deficits in schizophrenia: toward a neurobiologically informed approach. Biol. Psychiatry 81, e1-e3 (2017).

12. Ohi, K. et al. Genetic overlap between general cognitive function and schizophrenia: a review of cognitive GWASs. Int. J. Mol. Sci. 19, 3822 (2018).

13. Seiler, N. et al. Prevalence of subthreshold positive symptoms in young people without psychotic disorders presenting to a youth mental health service. Schizophr. Res. 215, 446-448 (2019).

14. Smith, M. J., Barch, D. M., Thompson, P. A. \& Csernansky, J. G. Subclinical expression of schizophrenia-like symptoms in non-psychotic siblings of individuals with schizophrenia. Schizophr. Res. 103, 324-325 (2008).

15. Ahmed, A. O., Strauss, G. P., Buchanan, R. W., Kirkpatrick, B. \& Carpenter, W. T. Schizophrenia heterogeneity revisited: Clinical, cognitive, and psychosocial correlates of statistically-derived negative symptoms subgroups. J. Psychiatr. Res. 97, 8-15 (2018).

16. Thompson, W. K. et al. Characterizing trajectories of cognitive functioning in older adults with schizophrenia: does method matter? Schizophr. Res. 143, 90-96 (2013).

17. Case, M. et al. The heterogeneity of antipsychotic response in the treatment of schizophrenia. Psychol. Med. 41, 1291-1300 (2011).

18. Lewandowski, K., Sperry, S., Cohen, B. \& Öngür, D. Cognitive variability in psychotic disorders: a cross-diagnostic cluster analysis. Psychol. Med. 44 3239-3248 (2014).

19. Austin, S. F. et al. Long-term trajectories of positive and negative symptoms in first episode psychosis: a 10year follow-up study in the OPUS cohort. Schizophr. Res. 168, 84-91 (2015).

20. Abdin, E. et al. Trajectories of positive, negative and general psychopathology symptoms in first episode psychosis and their relationship with functioning over a 2-year follow-up period. PloS ONE 12, e0187141 (2017).

21. Craddock, K. E. S. et al. Symptom dimensions and subgroups in childhoodonset schizophrenia. Schizophr. Res. (2017).

22. Levine, S. Z. \& Rabinowitz, J. Trajectories and antecedents of treatment response over time in early-episode psychosis. Schizophr. Bull. 36, 624-632 (2010).

23. Waters, F. \& Fernyhough, C. Hallucinations: a systematic review of points of similarity and difference across diagnostic classes. Schizophr. Bull. 43, 32-43 (2017).

24. Buchanan, R. W. Persistent negative symptoms in schizophrenia: an overview. Schizophr. Bull. 33, 1013-1022 (2006).

25. Mäkinen, J., Miettunen, J., Isohanni, M. \& Koponen, H. Negative symptoms in schizophrenia-a review. Nord. J. Psychiatry 62, 334-341 (2008).

26. Boutros, N. N., Mucci, A., Diwadkar, V. \& Tandon, R. Negative symptoms in schizophrenia: a comprehensive review of electrophysiological investigations. Clin. schizophrenia Relat. Psychoses 8, 28-35B (2013).

27. Szoke, A. et al. Longitudinal studies of cognition in schizophrenia: metaanalysis. Br. J. Psychiatry 192, 248-257 (2008).

28. Alfimova, M. V., Kondratiev, N. V. \& Golimbet, V. E. Results and promises of genetics of cognitive impairment in schizophrenia: molecular-genetic approaches. Zh. Nevrol. Psikhiatr. Im. S. S. Korsakova 116, 137-144 (2016).

29. Misiak, B. et al. Cytokines and C-reactive protein alterations with respect to cognitive impairment in schizophrenia and bipolar disorder: a systematic review. Schizophr. Res. 192, 16-29 (2018)

30. Bortolato, B., Miskowiak, K. W., Kohler, C. A., Vieta, E. \& Carvalho, A. F. Cognitive dysfunction in bipolar disorder and schizophrenia: a systematic review of meta-analyses. Neuropsychiatr. Dis. Treat. 11, 3111-3125 (2015).

31. Mesholam-Gately, R. I., Giuliano, A. J., Goff, K. P., Faraone, S. V. \& Seidman, L. J. Neurocognition in first-episode schizophrenia: a meta-analytic review. Neuropsychology 23, 315-336 (2009).

32. Snitz, B. E., MacDonald, A. W. III \& Carter, C. S. Cognitive deficits in unaffected first-degree relatives of schizophrenia patients: a meta-analytic review of putative endophenotypes. Schizophr. Bull. 32, 179-194 (2005).

33. Bozikas, V. P. \& Andreou, C. Longitudinal studies of cognition in first episode psychosis: a systematic review of the literature. Aust. N. Z. J. Psychiatry 45 93-108 (2011).

34. Ventura, J., Wood, R. C. \& Hellemann, G. S. Symptom domains and neurocognitive functioning can help differentiate social cognitive processes in schizophrenia: a meta-analysis. Schizophr. Bull. 39, 102-111 (2011).

35. Fett, A. J. et al. The relationship between neurocognition and social cognition with functional outcomes in schizophrenia: a meta-analysis. Neurosci. Biobehav. Rev. 35, 573-588 (2011).
36. Schober, P., Boer, C. \& Schwarte, L. A. Correlation coefficients: appropriate use and interpretation. Anesthesia Analgesia 126, 1763-1768 (2018).

37. Xavier, R. M. \& Vorderstrasse, A. Genetic basis of positive and negative symptom domains in schizophrenia. Biol. Res. Nurs. 19, 559-575 (2017)

38. Sauvé, G., Malla, A., Joober, R., Brodeur, M. B. \& Lepage, M. Comparing cognitive clusters across first-and multiple-episode of psychosis. Psychiatry Res. 269, 707-718 (2018).

39. Habtewold, T. D., Liemburg, E. J., Bruggeman, R., \& Alizadeh, B. Z. Symptomatic trajectories and clusters in patients with schizophrenia, siblings and healthy controls. https://www.crd.york.ac.uk/prospero/display_record.php? ID $=$ CRD42018093566 (2019)

40. Moher, D., Liberati, A., Tetzlaff, J. \& Altman, D. G. \& Prisma Group. Preferred reporting items for systematic reviews and meta-analyses: the PRISMA statement. PLoS Med. 6, 1-6 (2009).

41. Beller, E. M. et al. PRISMA for abstracts: reporting systematic reviews in journal and conference abstracts. PLoS Med. 10, e1001419 (2013).

42. Frankfurt, S., Frazier, P., Syed, M. \& Jung, K. R. Using group-based trajectory and growth mixture modeling to identify classes of change trajectories. Couns. Psychol. 44, 622-660 (2016).

43. Guyatt, G. H. et al. GRADE: an emerging consensus on rating quality of evidence and strength of recommendations. BMJ 336, 924-926 (2008).

44. Cheah, S.- et al. Interaction of multiple gene variants and their effects on schizophrenia phenotypes. Compr. Psychiatry 71, 63-70 (2016).

45. Cocchi, A. et al. Patients with first-episode psychosis are not a homogeneous population: Implications for treatment. Clin. Pract. Epidemiol. Ment. Health 10 1-8 (2014).

46. Hall, M.- et al. Patterns of deficits in brain function in bipolar disorder and schizophrenia: a cluster analytic study. Psychiatry Res. 200, 272-280 (2012).

47. Kavanaugh, B. C. et al. Neurocognitive phenotypes in severe childhood psychiatric disorders. J. Nerv. Ment. Dis. 204, 770-777 (2016).

48. Lim, J. et al. Elucidation of shared and specific white matter findings underlying psychopathology clusters in schizophrenia. Asian J. Psychiatry $\mathbf{3 0}$ 144-151 (2017).

49. Lin, S.- et al. Clustering by neurocognition for fine mapping of the schizophrenia susceptibility loci on chromosome 6p. Genes Brain Behav. 8, 785-794 (2009).

50. Nordon, C. et al. Trajectories of antipsychotic response in drug-naive schizophrenia patients: results from the 6-month ESPASS follow-up study. Acta Psychiatr. Scand. 129, 116-125 (2014).

51. Silver, H. \& Shmoish, M. Analysis of cognitive performance in schizophrenia patients and healthy individuals with unsupervised clustering models. Psychiatry Res. 159, 167-179 (2008)

52. Islam, M. A. et al. Long-term cognitive trajectories and heterogeneity in patients with schizophrenia and their unaffected siblings. Acta Psychiatr. Scand. 138, 591-604 (2018).

53. Ohi, K. et al. Cognitive clustering in schizophrenia patients, their first-degree relatives and healthy subjects is associated with anterior cingulate cortex volume. Neuroimage Clin. 16, 248-256 (2017).

54. Quee, P. J., Alizadeh, B. Z., Aleman, A. \& van den Heuvel, E. \& GROUP Investigators. Cognitive subtypes in non-affected siblings of schizophrenia patients: characteristics and profile congruency with affected family members. Psychol. Med. 44, 395-405 (2014)

55. Lui, S. S. Y. et al. Clustering of schizotypal features in unaffected first-degree relatives of schizophrenia patients. Schizophr. Bull. 44, S536-S546 (2018).

56. Stauffer, $V$. et al. Trajectories of response to treatment with atypical antipsychotic medication in patients with schizophrenia pooled from 6 doubleblind, randomized clinical trials. Schizophr. Res. 130, 11-19 (2011).

57. Chen, L. et al. The longitudinal interplay between negative and positive symptom trajectories in patients under antipsychotic treatment: a post hoc analysis of data from a randomized, 1-year pragmatic trial. BMC Psychiatry. 13 320 (2013)

58. Chang, W. C. et al. Early-stage negative symptom trajectories and relationships with 13-year outcomes in first-episode nonaffective psychosis. Schizophr. Bull. 45, 610-619 (2018).

59. Jäger, $M$. et al. Identification of psychopathological course trajectories in schizophrenia. Psychiatry Res. 215, 274-279 (2014).

60. Schennach, R. et al. Response trajectories in "real-world" naturalistically treated schizophrenia patients. Schizophr. Res. 139, 218-224 (2012).

61. Gee, B. et al. The course of negative symptom in first episode psychosis and the relationship with social recovery. Schizophr. Res. 174, 165-171 (2016) 
62. Levine, S. Z., Rabinowitz, J., Case, M. \& Ascher-Svanum, H. Treatment response trajectories and their antecedents in recent-onset psychosis: a 2-year prospective study. J. Clin. Psychopharmacol. 30, 446-449 (2010).

63. Levine, S. Z., Rabinowitz, J., Faries, D., Lawson, A. H. \& Ascher-Svanum, H. Treatment response trajectories and antipsychotic medications: examination of up to 18 months of treatment in the CATIE chronic schizophrenia trial. Schizophr. Res. 137, 141-146 (2012).

64. Stiekema, A. P. et al. Long-term course of negative symptom subdomains and relationship with outcome in patients with a psychotic disorder. Schizophr. Res. 193, 173-181 (2017).

65. Pelayo-Teran, J. et al. Trajectories of symptom dimensions in short-term response to antipsychotic treatment in patients with a first episode of nonaffective psychosis. Psychol. Med. 44, 37-50 (2014).

66. Habtewold, T. D. et al. Association of schizophrenia polygenic risk score with data-driven cognitive subtypes: a six-year longitudinal study in patients, siblings and controls. Schizophr. Res. https://doi.org/10.1016/j. schres.2020.05.020 (2020).

67. Chan, S. K. W. et al. Ten-year trajectory and outcomes of negative symptoms of patients with first-episode schizophrenia spectrum disorders. Schizophr. Res. 220, 85-91 (2020).

68. Wang, Y. et al. Trajectories of schizotypy and their emotional and social functioning: An 18-month follow-up study. Schizophr. Res. 193, 384-390 (2018).

69. Trauelsen, A. M. et al. Metacognition in first-episode psychosis and its association with positive and negative symptom profiles. Psychiatry Res. $\mathbf{2 3 8}$ 14-23 (2016).

70. Potter, A. I. \& Nestor, P. G. IQ subtypes in schizophrenia: distinct symptom and neuropsychological profiles. J. Nerv. Ment. Dis. 198, 580-585 (2010)

71. Ochoa, S. et al. Cognitive profiles of three clusters of patients with a firstepisode psychosis. Schizophr. Res. 150, 151-156 (2013).

72. Wang, Y., Neumann, D., Shum, D. H. K. \& Chan, R. C. K. A cross-validation study of clustering of schizotypy using a non-clinical Chinese sample. Psychiatry Res. 200, 55-58 (2012).

73. Barrantes-Vidal, N., Lewandowski, K. E. \& Kwapil, T. R. Psychopathology, social adjustment and personality correlates of schizotypy clusters in a large nonclinical sample. Schizophr. Res. 122, 219-225 (2010).

74. Lysaker, P. H., Vohs, J. L. \& Tsai, J. Negative symptoms and concordant impairments in attention in schizophrenia: associations with social functioning, hope, self-esteem and internalized stigma. Schizophr. Res. 110 165-172 (2009).

75. Geisler, D. et al. Brain structure and function correlates of cognitive subtypes in schizophrenia. Psychiatry Res. 234, 74-83 (2015).

76. Bell, M. D., Johannesen, J. K., Greig, T. C. \& Wexler, B. E. Memory profiles in schizophrenia: categorization validity and stability. Schizophr. Res. 118, 26-33 (2010).

77. Talpalaru, A., Bhagwat, N., Devenyi, G. A., Lepage, M. \& Chakravarty, M. M. Identifying schizophrenia subgroups using clustering and supervised learning. Schizophr. Res. 214, 51-59 (2019).

78. Wu, M., Chan, F., Wang, T.- \& Chen, S.- Neurocognitive profiles of rehabilitation clients with schizophrenia in taiwan. J. Rehabil. 76, 10-14 (2010).

79. Rodriguez, M. et al. Cluster analysis and correlations between cognitive domains: Cognitive performance in a Czech sample of first episodes schizophrenia spectrum disorders-preliminary results. Psychiatrie 21, 4-11 (2017).

80. Prouteau, A., Roux, S., Destaillats, J.- \& Bergua, V. Profiles of relationships between subjective and objective cognition in schizophrenia: Associations with quality of life, stigmatization, and mood factors. J. Cogn. Educ. Psychol. 16, 64-76 (2017)

81. Crouse, J. J., Moustafa, A. A., Bogaty, S. E., Hickie, I. B. \& Hermens, D. F. Parcellating cognitive heterogeneity in early psychosis-spectrum illnesses: a cluster analysis. Schizophr. Res. 202, 91-98 (2018).
82. Gilbert, E. et al. Cluster analysis of cognitive deficits may mark heterogeneity in schizophrenia in terms of outcome and response to treatment. Eur. Arch. Psychiatry Clin. Neurosci. 264, 333-343 (2014).

83. Chang, J. S. et al. Differences in the internal structure of hallucinatory experiences between clinical and nonclinical populations. Psychiatry Res. 226, 204-210 (2015).

84. Uren, J., Cotton, S. M., Killackey, E., Saling, M. M. \& Allott, K. Cognitive clusters in first-episode psychosis: overlap with healthy controls and relationship to concurrent and prospective symptoms and functioning. Neuropsychology $\mathbf{3 1}$, 787-797 (2017).

85. Strauss, G. P. et al. Deconstructing negative symptoms of schizophrenia: Avolition-apathy and diminished expression clusters predict clinical presentation and functional outcome. J. Psychiatr. Res. 47, 783-790 (2013).

86. Reser, M. P., Allott, K. A., Killackey, E., Farhall, J. \& Cotton, S. M. Exploring cognitive heterogeneity in first-episode psychosis: What cluster analysis can reveal. Psychiatry Res. 229, 819-827 (2015).

87. Lewandowski, K. E., Baker, J. T., McCarthy, J. M., Norris, L. A. \& Öngür, D. Reproducibility of cognitive profiles in psychosis using cluster analysis. J. Int. Neuropsychol. Soc. 24, 382-390 (2018).

88. Dawes, S. E., Jeste, D. V. \& Palmer, B. W. Cognitive profiles in persons with chronic schizophrenia. J. Clin. Exp. Neuropsychol. 33, 929-936 (2011).

89. Bell, M. D., Corbera, S., Johannesen, J. K., Fiszdon, J. M. \& Wexler, B. E. Social cognitive impairments and negative symptoms in schizophrenia: are there subtypes with distinct functional correlates? Schizophr. Bull. 39, 186-196 (2013).

90. Smucny, J. et al. Latent profiles of cognitive control, episodic memory, and visual perception across psychiatric disorders reveal a dimensional structure. Schizophr. Bull. 46, 154-162 (2019).

91. Rangel, A. et al. Neurocognitive subtypes of schizophrenia. Actas Esp. Psiquiatr 43, 80-90 (2015)

92. Dickinson, D. et al. Distinct polygenic score profiles in schizophrenia subgroups with different trajectories of cognitive development. Am. J. Psychiatry, Appl. 2019, 19050527 (2019).

93. Bechi, M. et al. Intellectual and cognitive profiles in patients affected by schizophrenia. J. Neuropsychol 13, 589-602 (2018).

94. Rocca, P. et al. Social cognition in people with schizophrenia: a clusteranalytic approach. Psychol. Med. 46, 2717-2729 (2016).

95. Wells, R. et al. The impact of premorbid and current intellect in schizophrenia: cognitive, symptom, and functional outcomes. NPJ Schizophr 1, 15043 (2015).

96. Twisk, J. \& Hoekstra, T. Classifying developmental trajectories over time should be done with great caution: a comparison between methods. J. Clin. Epidemiol. 65, 1078-1087 (2012)

97. Muthén, B. \& Shedden, K. Finite mixture modeling with mixture outcomes using the EM algorithm. Biometrics 55, 463-469 (1999).

98. Marquand, A. F., Wolfers, T., Mennes, M., Buitelaar, J. \& Beckmann, C. F. Beyond lumping and splitting: a review of computational approaches for stratifying psychiatric disorders. Biol. Psychiatry. Cogn. Neurosci. Neuroimaging 1, 433-447 (2016).

99. Stroebe, W. \& Strack, F. The alleged crisis and the illusion of exact replication. Perspect. Psychol. Sci. 9, 59-71 (2014).

100. Fusar-Poli, P. et al. Development and validation of a clinically based risk calculator for the transdiagnostic prediction of psychosis. JAMA Psychiatry $\mathbf{7 4}$ 493-500 (2017).

101. Greenwood, T. A., Shutes-David, A. \& Tsuang, D. W. Endophenotypes in schizophrenia: digging deeper to identify genetic mechanisms. J. Psychiatry Brain Sci. 4, e190005 (2019)

102. Peter F. B. \& Brian J. M. Personalized medicine for schizophrenia. npj Schizophr. 3, https://doi.org/10.1038/s41537-016-0001-5 (2017) 